\title{
VARIATIONAL MULTISCALE FINITE ELEMENT METHOD FOR FLOWS IN HIGHLY POROUS MEDIA
}

\author{
O. ILIEV*, R. LAZAROV ${ }^{\dagger}$, AND J. WILLEMS ${ }^{\ddagger}$
}

\begin{abstract}
We present a two-scale finite element method for solving Brinkman's and Darcy's equations. These systems of equations model fluid flows in highly porous and porous media, respectively. The method uses a recently proposed discontinuous Galerkin FEM for Stokes' equations by Wang and Ye and the concept of subgrid approximation developed by Arbogast for Darcy's equations. In order to reduce the "resonance error" and to ensure convergence to the global fine solution the algorithm is put in the framework of alternating Schwarz iterations using subdomains around the coarse-grid boundaries. The discussed algorithms are implemented using the Deal.II finite element library and are tested on a number of model problems.
\end{abstract}

Key words. numerical upscaling, flow in heterogeneous porous media, Brinkman equations, Darcy's law, subgrid approximation, discontinuous Galerkin mixed FEM

AMS subject classifications. 80M40, 80M35, 35J25, 35R05, 76M50

1. Introduction. Flows in porous media appear in many industrial, scientific, engineering, and environmental applications. One common characteristic of these diverse areas is that porous media are intrinsicly multiscale and typically display heterogeneities over a wide range of length-scales. Depending on the goals, solving the governing equations of flows in porous media might be sought at:

(a) A coarse scale (e.g., if only the global pressure drop for a given flow rate is needed, and no other fine scale details of the solution are important).

(b) A coarse scale enriched with some desirable fine scale details.

(c) The fine scale (if computationally affordable and practically desirable).

At pore level slow flows of incompressible fluids through the connected network of pores are governed by Stokes' equations. On a field-level fluid flows in porous media have been modeled mainly by mass conservation equation and by Darcy's relation between the macroscopic pressure $p$ and velocity $\boldsymbol{u}$ :

$$
\nabla p=-\mu \kappa^{-1} \boldsymbol{u}
$$

with $\kappa$ being the permeability tensor and $\mu$ the viscosity.

In naturally occurring materials, e.g. soil or rock, the permeability is small in granite formations (say $10^{-15} \mathrm{~cm}^{2}$ ), medium in oil reservoirs, (say $10^{-7} \mathrm{~cm}^{2}$ to $10^{-9} \mathrm{~cm}^{2}$ ), and large in highly fractured or in vuggy media (say $10^{-3} \mathrm{~cm}^{2}$ ). The latter is characterized by a high porosity. Aside from these examples from hydrology and geoscience there are also numerous instances of highly porous man-made materials, which are important for the engineering practice. These examples include mineral wool with porosity up to $99.7 \%$ (see Figure 1.1(a)) and industrial foams with porosity up to 95\% (see Figure 1.1(b)).

In order to reduce the deviations between the measurements for flows in highly porous media, such as the ones just mentioned, and the Darcy-based predictions, Brinkman in [9]

\footnotetext{
* Fraunhofer Institut für Techno- und Wirtschaftsmathematik, Fraunhofer-Platz 1, 67663 Kaiserslautern, Germany and Institute of Mathematics and Informatics, Bulg. Acad. Sci., Acad. G. Bonchev str., bl. 8, 1113 Sofia, Bulgaria (oleg.iliev@itwm. fraunhofer.de).

${ }^{\dagger}$ Dept. Mathematics, Texas A\&M University, College Station, Texas, 77843, USA and Institute of Mathematics and Informatics, Bulg. Acad.Sci., Acad. G. Bonchev str., bl. 8, 1113 Sofia, Bulgaria (lazarov@math.tamu.edu)

${ }^{\ddagger}$ Dept. Mathematics, Texas A\&M University, College Station, Texas, 77843, USA (jwillems@math.tamu . edu)
} 
introduced a new phenomenological relation between the velocity and the pressure gradient (see, also [23, page 94]):

$$
\nabla p=-\mu \kappa^{-1} \boldsymbol{u}+\mu \Delta \boldsymbol{u} .
$$

Darcy's and Brinkman's relations are then augmented by proper boundary conditions and by the conservation of mass principle, which in the absence of any mass sources/sinks is expressed by $\nabla \cdot \boldsymbol{u}=0$. Solving the corresponding equations in heterogeneous media is a difficult task which up to now is not fully mastered.

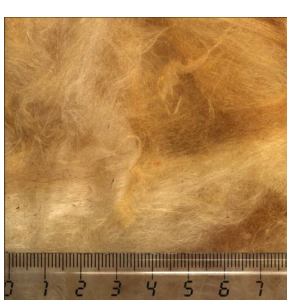

(a) Glass wool: macro- and microstructures

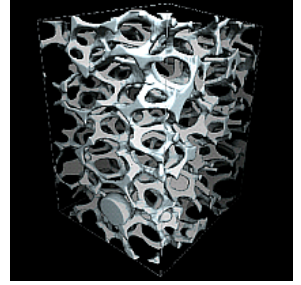

(b) Industrial open foams on a microstructure scale

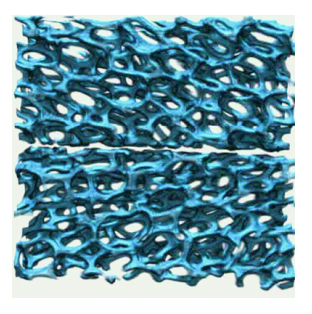

(1)

FIG. 1.1. Highly porous materials on a macro- and micro-scales

Darcy's and Brinkman's equations were introduced as phenomenological macroscopic equations without direct link to underlying microscopic behavior. Nevertheless, advances in homogenization theory made it possible to rigorously derive Darcy's and Brinkman's equations from Stokes' equations. The case of slow viscous fluid flow at pore level, when slip effects at interfaces between the fluid and solid walls are negligible, was extensively studied for periodic geometries, see e.g. [1, 19, 30]. As concluded in [1, pp. 266-273], there are three different limits depending on the size of the periodically arranged obstacles, which respectively lead to Darcy's, Brinkman's, and Stokes' equations as macroscopic, i.e., homogenized, relation. In the case of rather simple geometries one may account for slip conditions at the interfaces between free and porous media flow regions by application of Beavers-Joseph-Saffman interface conditions (see e.g. [5, 21, 26]). For viscous flows in highly heterogeneous (and thus topologically complicated) and highly porous media like the ones mentioned above Brinkman's equations are considered to be an adequate model (cf. $[9,7,32])$.

Brinkman's system of equations is part of a large class of mathematical problems describing various types of flows of compressible and incompressible fluids treated by the fictitious regions method. These include flows in porous media, [25], time dependent incompressible viscous flows, [10, 16, 24], transient compressible viscous flows, [33, 36]. The rigorous analysis of Brinkman's system from the point of view fictitious domain method was carried out in [2]. This formulation is used to replace Stokes flow in a complicated domain (flow around many obstacles, or an obstacle with complicated topology, e.g. Figure 1.1(b)), with Brinkman's equations in a simpler domain but with highly varying coefficients. Note, that in the literature concerning fictitious region (fictitious domain) methods for flow problems, this system does not have an established name. Although, sometimes it is called perturbed Stokes's system, in this paper we will refer to it as Brinkman's system.

Summing up, there is an abundance of challenging multiscale problems in physics and engineering modeled by Brinkman's equations at micro-, meso-, or macro-scale.

Motivated by these practical applications in this paper we consider numerical methods and solution techniques for porous media flows in both Brinkman and Darcy regimes. More precisely, we have the following specific goals: 
1. Devise a subgrid (variational multiscale) method for Brinkman's problem allowing to compute a two-scale (enriched coarse scale) solution, case (b) above;

2. Derive a subgrid based two level domain decomposition method for solving Brinkman's problem in highly heterogeneous porous media at fine scale (sometimes called iterative upscaling), see case (c) above, and also devise such method for Darcy's problem as well;

3. Give a unified framework of the subgrid method for both Darcy's and Brinkman's problem.

According to the first goal in this article we derive and study a two-scale finite element method for Brinkman's equations using the idea of subgrid (variational multiscale) methods earlier developed by Arbogast for Darcy's problem (cf. [3]). The function $\kappa$ may represent permeability variations involving different length-scales, see, e.g. Figure 1.1 (see also [31]). To the best of our knowledge, there is no subgrid method for Brinkman's problem in the literature, except the short announcement in our earlier publications (cf. [20, 40]).

The discretization of (2.1) is based on a Discontinuous Galerkin (DG) finite element method using $H(d i v)$-conforming velocity functions. This method has been proposed by Wang and Ye (cf. [39]) to approximate Stokes equations. For details concerning this extension we refer to [40]. A discretization of Brinkman's equation using $H^{1}$-conforming elements that works well in the Darcy limit was proposed in [18]. The reasons for adopting the discontinuous Galerkin method are:

- optimal orders of convergence in the Stokes and Darcy limiting regimes

- additional crucial properties of the mixed finite element spaces (see (2.4)), necessary for the derivation of the numerical subgrid method.

- local mass conservation ensured by piecewise constant pressure functions.

According to the second goal in this article we extend the subgrid approximations to numerically treat problems without scale separations. More precisely, by enhancing the method with overlapping subdomains we devise an alternating Schwarz method for computing the fine grid approximate solution. Similar multiscale Domain Decomposition methods for Darcy's problem have been presented earlier in connection with multiscale finite element, [13, 14, 15], energy minimizing basis functions, [37, 42, 38], etc. To the best of our knowledge, there has been no multiscale domain decomposition method for Brinkman's equation and/or variational multiscale (VMS) based domain decomposition method for Darcy's problem.

The subgrid method for Brinkman's and for Darcy's problem is presented in a unified way, which allows to identify the similarities and the differences of the variational multiscale approach for these two problems. Morever, this method works rather well in both limiting cases, Stokes and Darcy. Recall that the VMS method for Darcy's problem was presented earlier, e.g., in $[3,29]$.

The remainder of this paper is organized as follows: In the next section we provide a detailed description of the problems under consideration as well as the necessary notation. Section 3 is devoted to the description of a DG discretization of Brinkman's equations. In section 4 we outline the derivation of the numerical subgrid algorithm for Brinkman's and Darcy's equations. After that we discuss an extension of this algorithm by alternating Schwarz iterations. The final section contains numerical experiments corresponding to the presented algorithms as well as conclusions.

2. Problem Formulation and Notation. We use the standard notation for spaces of scalar and vector-valued functions defined on a bounded simply connected domain $\Omega \subset$ $\mathbb{R}^{n}(n=2,3)$ with polyhedral boundary having the outward unit normal vector $\boldsymbol{n}$. Further, $L_{0}^{2}(\Omega) \subset L^{2}(\Omega)$ is the space of square integrable functions with mean value zero and 
$H^{1}(\Omega)^{n}, H_{0}^{1}(\Omega)^{n}$, and $L^{2}(\Omega)^{n}$ denote the spaces of vector-valued functions with components in $H^{1}(\Omega), H_{0}^{1}(\Omega)$, and $L^{2}(\Omega)$, respectively. Furthermore,

$$
\begin{gathered}
H(\operatorname{div} ; \Omega):=\left\{\boldsymbol{v} \in L^{2}(\Omega)^{n}: \nabla \cdot \boldsymbol{v} \in L^{2}(\Omega)\right\}, \\
H_{0}(\operatorname{div} ; \Omega):=\{\boldsymbol{v} \in H(\operatorname{div} ; \Omega): \boldsymbol{v} \cdot \boldsymbol{n}=0 \text { on } \partial \Omega\},
\end{gathered}
$$

equipped with the norm

$$
\|\boldsymbol{v}\|_{H(d i v ; \Omega)}=\left(\int_{\Omega}\left(|\nabla \cdot \boldsymbol{v}|^{2}+|\boldsymbol{v}|^{2}\right) d \boldsymbol{x}\right)^{\frac{1}{2}}
$$

and where the values at the boundary are assumed in the usual trace sense. We also use the standard notation $\nabla \boldsymbol{u}: \nabla \boldsymbol{v}:=\sum_{i, k=1}^{n} \frac{\partial u_{i}}{\partial x_{k}} \frac{\partial v_{i}}{\partial x_{k}}$, where $\boldsymbol{u}=\left(u_{1}, \ldots, u_{n}\right)$ and $\boldsymbol{v}=\left(v_{1}, \ldots, v_{n}\right)$. Further, we denote by $P_{k}$ the space of polynomials of degree $k \in \mathbb{N}_{0}$ and, consistently with our notation, $P_{k}^{n}$ denotes the set of vector-valued functions having $n$ components in $P_{k}$.

As mentioned in the introduction, our work is dedicated to the numerical upscaling of Brinkman's and Darcy's equations:

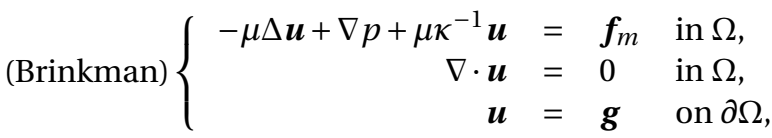

$$
\begin{aligned}
& \text { (Darcy) }\left\{\begin{aligned}
\nabla p+\mu \kappa^{-1} \boldsymbol{u} & =\boldsymbol{f}_{m} & & \text { in } \Omega, \\
\nabla \cdot \boldsymbol{u} & =0 & & \text { in } \Omega, \\
\boldsymbol{u} \cdot \boldsymbol{n} & = & & \text { on } \partial \Omega,
\end{aligned}\right.
\end{aligned}
$$

where the viscosity $\mu$ is assumed to be a positive constant, the permeability $\kappa \in L^{\infty}(\Omega)$ with $\infty>\kappa_{\text {max }} \geq \kappa \geq \kappa_{\text {min }}>0, \boldsymbol{f}_{m} \in L^{2}(\Omega)^{n}$ is a forcing term ( $m$ stands for "momentum"), and the boundary data $g \in H^{\frac{1}{2}}(\partial \Omega)^{n}$ and $g \in H^{\frac{1}{2}}(\partial \Omega)$ satisfy the compatibility condition

$$
\int_{\partial \Omega} \boldsymbol{g} \cdot \boldsymbol{n} d s=0 \text { and } \int_{\partial \Omega} g d s=0
$$

respectively. With these assumptions problems (2.1) and (2.2) have unique weak solutions $(\boldsymbol{u}, p)$ in $\left(H^{1}(\Omega)^{n}, L_{0}^{2}(\Omega)\right)$ and $\left(H(\operatorname{div} ; \Omega), L_{0}^{2}(\Omega)\right)$, respectively. The smoothness of the velocity solutions of these problems can be studied by the methods developed in [12, 17]. We shall assume that $\boldsymbol{u} \in\left(H^{s}(\Omega)\right)^{n}$ with some $s>\frac{3}{2}$, where $H^{s}(\Omega)$, for noninteger $s$ is the standard interpolation space.

To make the derivation of the numerical subgrid upscaling method more transparent we adopt a semi-discrete setting. More specifically, we assume that all "coarse global" problems are posed with respect to a (finite dimensional) finite element space, whereas all "fine local" problems are solved exactly in an infinite dimensional space. In practical computations, we can only approximate the fine local problems by finite dimensional ones based on a finite element partition of each coarse-grid cell. Nevertheless, for the presentation of the method this setting greatly simplifies the exposition.

We need the following notation. Let $\mathscr{T}$ be a quasi-uniform partition of $\Omega$ into parallelepipeds of size $H$. Let $\mathscr{I}$ denote the set of all edges/faces of $\mathscr{T}$. Also, we define $\mathscr{I}$ to be

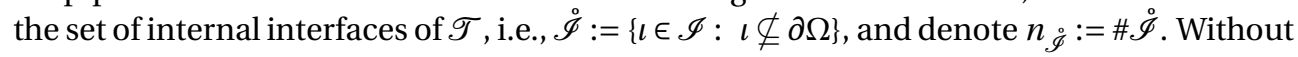




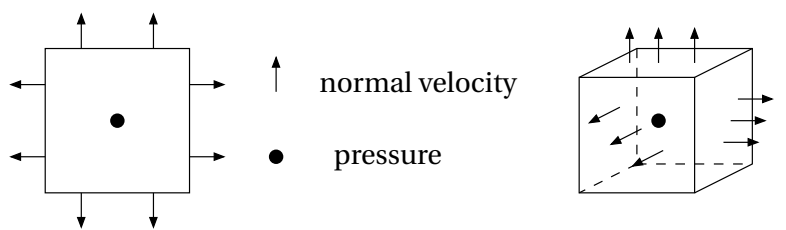

FIG. 2.1. Degrees of freedom of the BDM1 finite element space.

loss of generality, we assume that the interfaces in $\stackrel{\circ}{\mathscr{I}}$ are numbered, i.e., $\stackrel{\mathscr{I}}{ }=\left\{\iota^{i}\right\}_{i=1 \ldots n_{\mathscr{I}}}$. Also, we denote the set of all boundary edges/faces by $\mathscr{I}^{\partial}$, i.e., $\mathscr{I}^{\partial}:=\mathscr{I} \backslash \mathscr{I}^{\circ}$. For each $\iota \in \mathscr{I}^{\circ}$ we also define $N(\iota)$ to be an $\mathscr{O}(H)$-neighborhood of $\iota$, i.e., $N(\iota):=\left\{\boldsymbol{x} \in \Omega: \operatorname{dist}(\boldsymbol{x}, \iota)<C_{l} H\right\}$. Here $C_{l}<1$ is a constant independent of $H$.

Now, let $\mathscr{V}_{H}^{\partial} \subset H($ div; $\Omega$ ) be the Brezzi-Douglas-Marini (BDM1) mixed finite element spaces of degree 1 with respect to $\mathscr{T}$ (cf. e.g. [8, pages 120-130]). On the reference cell $(0,1)^{n}$ the space is characterized by

$$
P_{1}^{2}+\operatorname{span}\left\{\operatorname{curl}\left(x_{1}^{2} x_{2}\right), \operatorname{curl}\left(x_{1} x_{2}^{2}\right\}=P_{1}^{2}+\operatorname{span}\left\{\left(x_{1}^{2},-2 x_{1} x_{2}\right),\left(2 x_{1} x_{2},-x_{2}^{2}\right)\right\}, n=2\right.
$$

and

$$
P_{1}^{3}+\operatorname{span}\left\{\operatorname{curl}\left(0,0, x_{1} x_{2}^{2}\right), \operatorname{curl}\left(x_{2} x_{3}^{2}, 0,0\right), \operatorname{curl}\left(0, x_{1}^{2} x_{2}, 0\right), \operatorname{curl}\left(P_{0}^{3} x_{1} x_{2} x_{3}\right)\right\}, n=3
$$

The degrees of freedom of the BDM1 velocity functions are given by $\int_{l} \boldsymbol{v} \cdot \boldsymbol{n} r d s$ with $r \in$ $P_{1}(\iota)$ on each edge/face $\iota$ of the reference cell. Furthermore, the normal component of $\boldsymbol{v}$ is restricted to be continuous across cell boundaries.

The pressure space $\mathscr{W}_{H} \subset L_{0}^{2}(\Omega)$ consists of piecewise constant functions (constant on each $T \in \mathscr{T}$ ). We refer to Figure 2.1 for an illustration of the degrees of freedom of the BDM1 element.

Additionally, we introduce the finite element space $\mathscr{V}_{H} \subset \mathscr{V}_{H}^{\partial}$ of functions in $\mathscr{V}_{H}^{\partial}$ whose normal traces vanish on $\partial \Omega$ so that $\mathscr{V}_{H} \subset H_{0}(\operatorname{div} ; \Omega)$.

In the following we treat the Brinkman and the Darcy case simultaneously by using a unified notation. For each $T \in \mathscr{T}$ and $\iota \in \stackrel{\mathscr{I}}{\text { let }}$

$$
(\delta \mathscr{V}(T), \delta \mathscr{W}(T))= \begin{cases}\left(H_{0}^{1}(T)^{n}, L_{0}^{2}(T)\right), & \text { in the Brinkman case } \\ \left(H_{0}(\operatorname{div} ; T), L_{0}^{2}(T)\right), & \text { in the Darcy case }\end{cases}
$$

and

$$
\left(\mathscr{V}^{\tau}(\iota), \mathscr{W}^{\tau}(\iota)\right)= \begin{cases}\left(H_{0}^{1}(N(\iota))^{n}, L_{0}^{2}(N(\iota))\right), & \text { in the Brinkman case } \\ \left(H_{0}(d i v ; N(\iota)), L_{0}^{2}(N(\iota))\right), & \text { in the Darcy case. }\end{cases}
$$

Recall, that above we have defined $N(\iota)$ to be an $\mathscr{O}(H)$-neighborhood of $\iota$. We also consider the (direct) sums of these local spaces and set

$$
(\delta \mathscr{V}, \delta \mathscr{W}):=\bigoplus_{T \in \mathscr{T}}(\delta \mathscr{V}(T), \delta \mathscr{W}(T))
$$

and

$$
\left(\mathscr{V}^{\tau}, \mathscr{W}^{\tau}\right):=\sum_{\iota \in \mathscr{I}}\left(\mathscr{V}^{\tau}(\iota), \mathscr{W}^{\tau}(\iota)\right)
$$


where functions in $(\delta \mathscr{V}(T), \delta \mathscr{W}(T))$ and $\left(\mathscr{V}^{\tau}(\iota), \mathscr{W}^{\tau}(\iota)\right)$ are extended by zero to $\Omega \backslash T$ and $\Omega \backslash N(\iota)$, respectively. With these definitions it is clear that the introduced function spaces satisfy the following properties:

$$
\begin{gathered}
\nabla \cdot \delta \mathscr{V} \subset \delta \mathscr{W} \text { and } \nabla \cdot \mathscr{V}_{H} \subset \mathscr{W}_{H}, \\
\delta \mathscr{W} \perp \mathscr{W}_{H} \quad \text { in the } L^{2} \text {-inner-product, }
\end{gathered}
$$

and

$$
\mathscr{V}_{H} \cap \delta \mathscr{V}=\{\mathbf{0}\}
$$

Due to $(2.4 \mathrm{~b})$ and $(2.4 \mathrm{c})$ the following direct sum is well-defined.

$$
\left(\mathscr{V}_{H, \delta}, \mathscr{W}_{H, \delta}\right):=\left(\mathscr{V}_{H}, \mathscr{W}_{H}\right) \oplus(\delta \mathscr{V}, \delta \mathscr{W})
$$

REMARK 2.1. The composite space $\mathscr{V}_{H, \delta}$ differs from $H_{0}^{1}(\Omega)^{n}$ and $H_{0}($ div; $\Omega)$, respectively, in particular in that the former has only (finitely many) "coarse" degrees of freedom across coarse interfaces, i.e., $\iota \in \mathscr{I}$.

REMARK 2.2. In practice $\delta \mathscr{V}$ and $\delta \mathscr{W}$ will be finite element spaces of vector and scalar functions, respectively, that satisfy the properties (2.4). Candidates for such spaces are Brezzi, Douglas, and Marini (BDMk) or Raviart-Thomas (RTk) spaces of degree $k \geq 1$, (see, [8, pages 120-130]). Since the coarse space consists of BDM1 finite elements, from an approximation point of view, it does not make sense to use finite elements of order higher than one on the fine mesh. In our implementation we use BDM1 finite elements on the fine mesh, as well.

3. Discontinuous Galerkin Discretization of Brinkman's Equations. In this section we present a DG discretization of Brinkman's equations and - continuing our unified notational setting - a standard discretization of Darcy's equations. The DG discretization of Brinkman's equations is an extension of the one introduced and studied for Stokes' equations by Wang and Ye [39]. We consider discretizations of (2.1) and (2.2) using the mixed finite element space $\left(\mathscr{V}_{H}, \mathscr{W}_{H}\right)$. Note, that $\left(\mathscr{V}_{H}, \mathscr{W}_{H}\right)$ is conforming for the Darcy but nonconforming in the Brinkman case. Following well-established approaches for the derivation and analysis of DG discretizations (cf. e.g. [34, 39]) and using a classical result from [8] we arrive at the following discretization of (2.1) and (2.2):

Find $\left(\boldsymbol{u}_{H}, p_{H}\right) \in\left(\mathscr{V}_{H}, \mathscr{W}_{H}\right)$ such that for all $\left(\boldsymbol{v}_{H}, q_{H}\right) \in\left(\mathscr{V}_{H}, \mathscr{W}_{H}\right)$

$$
\left\{\begin{aligned}
a\left(\boldsymbol{u}_{H}, \boldsymbol{v}_{H}\right)+b\left(\boldsymbol{v}_{H}, p_{H}\right) & =F_{m}\left(\boldsymbol{v}_{H}\right), \\
b\left(\boldsymbol{u}_{H}, q_{H}\right) & =F_{s}\left(q_{H}\right),
\end{aligned}\right.
$$

where for $\boldsymbol{v}, \boldsymbol{w} \in \mathscr{V}_{H}$ the bilinear form $a(\cdot, \cdot)$ is defined as

$$
\begin{array}{cc}
a(\boldsymbol{w}, \boldsymbol{v}):=\left\{\begin{array}{cc}
a_{S}(\boldsymbol{w}, \boldsymbol{v})+a_{D}(\boldsymbol{w}, \boldsymbol{v})+a_{\mathscr{I}}(\boldsymbol{w}, \boldsymbol{v}) & \text { Brinkman } \\
a_{D}(\boldsymbol{w}, \boldsymbol{v}) & \text { Darcy }
\end{array}\right. \\
a_{S}(\boldsymbol{w}, \boldsymbol{v}):=\mu \sum_{T \in \mathscr{T}} \int_{T} \nabla \boldsymbol{w}: \nabla \boldsymbol{v} d \boldsymbol{x}, \quad a_{D}(\boldsymbol{w}, \boldsymbol{v}):=\mu \int_{\Omega} \kappa^{-1} \boldsymbol{w} \cdot \boldsymbol{v} d \boldsymbol{x},
\end{array}
$$




$$
\begin{gathered}
b(\boldsymbol{v}, q):=-\int_{\Omega} \nabla \cdot \boldsymbol{v} q d \boldsymbol{x} \\
F_{m}(\boldsymbol{v}):= \begin{cases}\left.\int_{\Omega} \boldsymbol{f} \cdot \boldsymbol{v} d \boldsymbol{x}+\mu \sum_{l \in \mathscr{I}^{\partial}} \int_{l}\left(\frac{\alpha}{H} \llbracket \boldsymbol{g} \rrbracket \cdot \llbracket \boldsymbol{v} \rrbracket-\llbracket \varepsilon(\boldsymbol{v})\right\} \cdot \llbracket \boldsymbol{g} \rrbracket\right) d s-a\left(\boldsymbol{u}_{\boldsymbol{g}}, \boldsymbol{v}\right) & \text { Brinkman } \\
\int_{\Omega}^{\boldsymbol{f}} \cdot \boldsymbol{v} d \boldsymbol{x}-a\left(\boldsymbol{u}_{\boldsymbol{g}}, \boldsymbol{v}\right) & \text { Darcy }\end{cases}
\end{gathered}
$$

and

$$
F_{s}(q):= \begin{cases}-b\left(\boldsymbol{u}_{\boldsymbol{g}}, q\right), & \text { Brinkman } \\ -b\left(\boldsymbol{u}_{g}, q\right), & \text { Darcy. }\end{cases}
$$

Above we use the following notation for the average of the normal derivative of the tangential velocity, $\{\varepsilon(\cdot)\}$, and the jump of the tangential component of the velocity, $\llbracket \cdot \rrbracket$ :

$$
\{\varepsilon(\boldsymbol{v})\}:= \begin{cases}\frac{1}{2}\left[\left(\nabla\left(\left.\boldsymbol{v}\right|_{T^{+}} \times \boldsymbol{n}^{+}\right)\right) \boldsymbol{n}^{+}+\left(\nabla\left(\left.\boldsymbol{v}\right|_{T^{-}} \times \boldsymbol{n}^{-}\right)\right) \boldsymbol{n}^{-}\right] & \text {on } \iota \in \dot{\mathscr{I}}, \\ \left(\nabla\left(\left.\boldsymbol{v}\right|_{T^{+}} \times \boldsymbol{n}^{+}\right)\right) \boldsymbol{n}^{+} & \text {on } \iota \in \mathscr{I}^{\partial}\end{cases}
$$

and

$$
\llbracket \boldsymbol{v} \rrbracket:= \begin{cases}\left.\boldsymbol{v}\right|_{T^{+}} \times \boldsymbol{n}^{+}+\left.\boldsymbol{v}\right|_{T^{-}} \times \boldsymbol{n}^{-} & \text {on } \iota \in \mathscr{I}, \\ \left.\boldsymbol{v}\right|_{T^{+}} \times \boldsymbol{n}^{+} & \text {on } \iota \in \mathscr{I}^{\partial},\end{cases}
$$

where as usual $\boldsymbol{n}$ denotes the outer unit normal vector. The superscripts ${ }^{+}$and ${ }^{-}$refer to the elements on either side of interface $\iota$.

In the Brinkman case the penalty parameter $\alpha \in \mathbb{R}^{+}$should be chosen sufficiently large (depending on the shape regularity of the underlying triangulation) in order for the bilinear form $a(\cdot, \cdot)$ to be positive definite.

We need to point out that the relations (3.5a) require a little bit more smoothness from the vector-function $v$ in order that the involved traces are well defined. However, in the subsequent implementation, these are piece-wise polynomial functions from certain finite element spaces and the traces are well defined.

Also, we assume that $\boldsymbol{u}_{\boldsymbol{g}} \in H^{1}(\Omega)$ and $\boldsymbol{u}_{\boldsymbol{g}} \in H($ div $: \Omega)$ are extensions of $\boldsymbol{g}$ and $g$, respectively, for which it holds that

$$
\boldsymbol{u}_{\boldsymbol{g}}, \boldsymbol{u}_{\mathrm{g}} \in \mathscr{V}_{H}^{\partial} .
$$

Thus, by the definition of $F_{s}(\cdot)$ we see that it is sufficient to consider the case of homogeneous boundary conditions, which we will henceforth assume.

Analogous to the analysis in [39] we obtain the following convergence result for the Brinkman case (for more details we also refer the reader to [40]).

THEOREM 3.1. Let $\left(\boldsymbol{u}_{H}, p_{H}\right)$ and $(\boldsymbol{u}, p)$ be the solution of (3.1) and (2.1), respectively. Assume also that $\boldsymbol{u}$ is $H^{2}(\Omega)$-regular and $p \in H^{1}(\Omega)$. Then there exists a constant $C$ independent of $H$ such that

$$
\left\|p-p_{H}\right\|_{L^{2}(\Omega)} \leq C H\left(\|\boldsymbol{u}\|_{H^{2}(\Omega)}+\|p\|_{H^{1}(\Omega)}\right)
$$

and

$$
\left\|\boldsymbol{u}-\boldsymbol{u}_{H}\right\|_{L^{2}(\Omega)} \leq C H^{2}\left(\|\boldsymbol{u}\|_{H^{2}(\Omega)}+\|p\|_{H^{1}(\Omega)}\right)
$$

REMARK 3.2. Here, it is worth noting that according to [39] and [8], respectively, analogous $L^{2}$-error estimates can be obtained when BDM1 finite elements are used for a DG discretization of Stokes equations and the classical discretization of Darcy's equations, respectively. 
4. Numerical Subgrid Method. We now outline the numerical subgrid approach for problems (2.1) and (2.2), which is essentially analogous to the method derived in [3, 4] for Darcy's equations. For this, we consider (3.1) posed with respect to the two-scale space $\left(\mathscr{V}_{H, \delta}, \mathscr{W}_{H, \delta}\right)$, i.e.: Find $\left(\boldsymbol{u}_{H, \delta}, p_{H, \delta}\right) \in\left(\mathscr{V}_{H, \delta}, \mathscr{W}_{H, \delta}\right)$ such that for all $\left(\boldsymbol{v}_{H, \delta}, q_{H, \delta}\right) \in\left(\mathscr{V}_{H, \delta}, \mathscr{W}_{H, \delta}\right)$ we have

$$
\left\{\begin{aligned}
a\left(\boldsymbol{u}_{H, \delta}, \boldsymbol{v}_{H, \delta}\right)+b\left(\boldsymbol{v}_{H, \delta}, p_{H, \delta}\right) & =F_{m}\left(\boldsymbol{v}_{H, \delta}\right) \\
b\left(\boldsymbol{u}_{H, \delta}, q_{H, \delta}\right) & =F_{S}\left(q_{H, \delta}\right)
\end{aligned}\right.
$$

Due to (2.5) we know that each function in $\left(\mathscr{V}_{H, \delta}, \mathscr{W}_{H, \delta}\right)$ may be uniquely decomposed into its components from $\left(\mathscr{V}_{H}, \mathscr{W}_{H}\right)$ and $(\delta \mathscr{V}, \delta \mathscr{W})$. Thus, (4.1) may be rewritten as

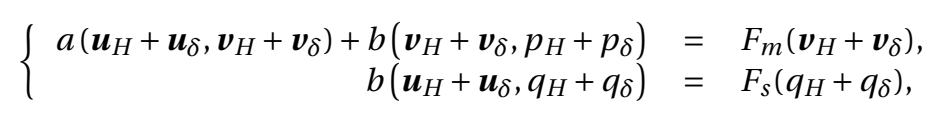

with $\boldsymbol{u}_{H, \delta}=\boldsymbol{u}_{H}+\boldsymbol{u}_{\delta}, \boldsymbol{v}_{H, \delta}=\boldsymbol{v}_{H}+\boldsymbol{v}_{\delta}, p_{H, \delta}=p_{H}+p_{\delta}$, and $q_{H, \delta}=q_{H}+q_{\delta}$, where $\boldsymbol{u}_{H}, \boldsymbol{v}_{H} \in \mathscr{V}_{H}$, $p_{H}, q_{H} \in \mathscr{W}_{H}, \boldsymbol{u}_{\delta}, \boldsymbol{v}_{\delta} \in \delta \mathscr{V}$, and $p_{\delta}, q_{\delta} \in \delta \mathscr{W}$. By linearity we may decompose (4.2) into

$$
\left\{\begin{aligned}
a\left(\boldsymbol{u}_{H}+\boldsymbol{u}_{\delta}, \boldsymbol{v}_{H}\right)+b\left(\boldsymbol{v}_{H}, p_{H}+p_{\delta}\right) & =F_{m}\left(\boldsymbol{v}_{H}\right) \forall \boldsymbol{v}_{H} \in \mathscr{V}_{H}, \\
b\left(\boldsymbol{u}_{H}+\boldsymbol{u}_{\delta}, q_{H}\right) & =F_{s}\left(q_{H}\right) \quad \forall q_{H} \in \mathscr{W}_{H}
\end{aligned}\right.
$$

and

$$
\left\{\begin{aligned}
a\left(\boldsymbol{u}_{H}+\boldsymbol{u}_{\delta}, \boldsymbol{v}_{\delta}\right)+b\left(\boldsymbol{v}_{\delta}, p_{H}+p_{\delta}\right) & =F_{m}\left(\boldsymbol{v}_{\delta}\right) \quad \forall \boldsymbol{v}_{\delta} \in \delta \mathscr{W} \\
b\left(\boldsymbol{u}_{H}+\boldsymbol{u}_{\delta}, q_{\delta}\right) & =F_{S}\left(q_{\delta}\right) \quad \forall q_{\delta} \in \delta \mathscr{W}
\end{aligned}\right.
$$

Due to (2.4a), (2.4b), (3.3), and (3.6) we may simplify (4.3) to obtain

$$
\left\{\begin{aligned}
a\left(\boldsymbol{u}_{H}+\boldsymbol{u}_{\delta}, \boldsymbol{v}_{H}\right)+b\left(\boldsymbol{v}_{H}, p_{H}\right) & =F_{m}\left(\boldsymbol{v}_{H}\right) \quad \forall \boldsymbol{v}_{H} \in \mathscr{V}_{H}, \\
b\left(\boldsymbol{u}_{H}, q_{H}\right) & =F_{s}\left(q_{H}\right) \quad \forall q_{H} \in \mathscr{W}_{H}
\end{aligned}\right.
$$

and

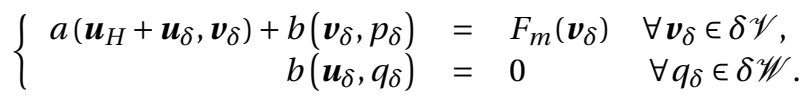

REMARK 4.1. This last step is actually crucial to ensure the solvability of (4.4b). In fact, the equivalence of (4.3b) and (4.4b) is a major reason for requiring properties (2.4) for the function spaces we use.

Now, by further decomposing $\left(\boldsymbol{u}_{\delta}, p_{\delta}\right)=\left(\boldsymbol{u}_{\delta}\left(F_{m}\right)+\boldsymbol{u}_{\delta}\left(\boldsymbol{u}_{H}\right), p_{\delta}\left(F_{m}\right)+p_{\delta}\left(\boldsymbol{u}_{H}\right)\right)$ and using superposition, (4.4b) may be replaced by the following systems of equations satisfied by $\left(\boldsymbol{u}_{\delta}\left(\boldsymbol{u}_{H}\right), p_{\delta}\left(\boldsymbol{u}_{H}\right)\right)$ and $\left(\boldsymbol{u}_{\delta}\left(F_{m}\right), p_{\delta}\left(F_{m}\right)\right)$, respectively:

$$
\left\{\begin{array}{rlll}
a\left(\boldsymbol{u}_{H}+\boldsymbol{u}_{\delta}\left(\boldsymbol{u}_{H}\right), \boldsymbol{v}_{\delta}\right)+b\left(\boldsymbol{v}_{\delta}, p_{\delta}\left(\boldsymbol{u}_{H}\right)\right) & = & 0 & \forall \boldsymbol{v}_{\delta} \in \delta \mathscr{V} \\
b\left(\boldsymbol{u}_{\delta}\left(\boldsymbol{u}_{H}\right), q_{\delta}\right) & = & 0 & \forall q_{\delta} \in \delta \mathscr{W}
\end{array}\right.
$$

and

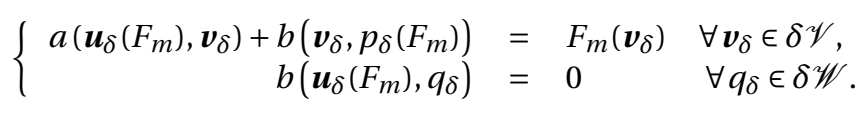

We easily see by (4.5a) that $\left(\boldsymbol{u}_{\delta}\left(\boldsymbol{u}_{H}\right), p_{\delta}\left(\boldsymbol{u}_{H}\right)\right)$ is a linear operator in $\boldsymbol{u}_{H}$. Note, that the solutions $\left(\boldsymbol{u}_{\delta}\left(F_{m}\right), p_{\delta}\left(F_{m}\right)\right)$ and for $\boldsymbol{u}_{H}$ given, $\left(\boldsymbol{u}_{\delta}\left(\boldsymbol{u}_{H}\right), p_{\delta}\left(\boldsymbol{u}_{H}\right)\right)$ can be computed locally due to the implicit homogeneous boundary condition in (2.3a), i.e., the restrictions of 
$\left(\boldsymbol{u}_{\delta}\left(F_{m}\right), p_{\delta}\left(F_{m}\right)\right)$ and $\left(\boldsymbol{u}_{\delta}\left(\boldsymbol{u}_{H}\right), p_{\delta}\left(\boldsymbol{u}_{H}\right)\right)$ to elements from $\mathscr{T}$ can be computed independently of each other. In the following we refer to $\left(\boldsymbol{u}_{\delta}\left(F_{m}\right), p_{\delta}\left(F_{m}\right)\right)$ and $\left(\boldsymbol{u}_{\delta}\left(\boldsymbol{u}_{H}\right), p_{\delta}\left(\boldsymbol{u}_{H}\right)\right)$ as the local responses to the right hand side and $\boldsymbol{u}_{H}$, respectively.

Plugging $\boldsymbol{u}_{\delta}\left(F_{m}\right)+\boldsymbol{u}_{\delta}\left(\boldsymbol{u}_{H}\right)$ into (4.4a) we arrive at the upscaled equation, which is entirely posed in terms of the coarse-grid unknowns, i.e.,

$$
\left\{\begin{aligned}
a\left(\boldsymbol{u}_{H}+\boldsymbol{u}_{\delta}\left(\boldsymbol{u}_{H}\right), \boldsymbol{v}_{H}\right)+b\left(\boldsymbol{v}_{H}, p_{H}\right) & =F_{m}\left(\boldsymbol{v}_{H}\right)-a\left(\boldsymbol{u}_{\delta}\left(F_{m}\right), \boldsymbol{v}_{H}\right) \\
b\left(\boldsymbol{u}_{H}, q_{H}\right) & =F_{S}\left(q_{H}\right)
\end{aligned}\right.
$$

Now, due to the first equation in (4.5a) we see by choosing $\boldsymbol{v}_{\delta}=\boldsymbol{u}_{\delta}\left(\boldsymbol{v}_{H}\right)$ that

$$
a\left(\boldsymbol{u}_{H}+\boldsymbol{u}_{\delta}\left(\boldsymbol{u}_{H}\right), \boldsymbol{u}_{\delta}\left(\boldsymbol{v}_{H}\right)\right)+b\left(\boldsymbol{u}_{\delta}\left(\boldsymbol{v}_{H}\right), p_{\delta}\left(\boldsymbol{u}_{H}\right)\right)=0 .
$$

The second equation in (4.5a) in turn yields $b\left(\boldsymbol{u}_{\delta}\left(\boldsymbol{v}_{H}\right), p_{\delta}\left(\boldsymbol{u}_{H}\right)\right)=0$. Combining these two results with (4.6) we obtain the symmetric upscaled system

$$
\left\{\begin{aligned}
a\left(\boldsymbol{u}_{H}+\boldsymbol{u}_{\delta}\left(\boldsymbol{u}_{H}\right), \boldsymbol{v}_{H}+\boldsymbol{u}_{\delta}\left(\boldsymbol{v}_{H}\right)\right)+b\left(\boldsymbol{v}_{H}, p_{H}\right) & =F_{m}\left(\boldsymbol{v}_{H}\right)-a\left(\boldsymbol{u}_{\delta}\left(F_{m}\right), \boldsymbol{v}_{H}\right) \forall \boldsymbol{v}_{H} \in \mathscr{V}_{H}, \\
b\left(\boldsymbol{u}_{H}, q_{H}\right) & =F_{S}\left(q_{H}\right) \forall q_{H} \in \mathscr{W}_{H} .
\end{aligned}\right.
$$

Now we define the symmetric bilinear form

$$
\widetilde{a}\left(\boldsymbol{u}_{H}, \boldsymbol{v}_{H}\right):=a\left(\boldsymbol{u}_{H}+\boldsymbol{u}_{\delta}\left(\boldsymbol{u}_{H}\right), \boldsymbol{v}_{H}+\boldsymbol{u}_{\delta}\left(\boldsymbol{v}_{H}\right)\right)
$$

so that the upscaled system can be rewritten in the form

$$
\left\{\begin{aligned}
\tilde{a}\left(\boldsymbol{u}_{H}, \boldsymbol{v}_{H}\right)+b\left(\boldsymbol{v}_{H}, p_{H}\right) & =F_{m}\left(\boldsymbol{v}_{H}\right)-a\left(\boldsymbol{u}_{\delta}\left(F_{m}\right), \boldsymbol{v}_{H}\right) \quad \forall \boldsymbol{v}_{H} \in \mathscr{V}_{H} \\
b\left(\boldsymbol{u}_{H}, q_{H}\right) & =F_{S}\left(q_{H}\right) \quad \forall q_{H} \in \mathscr{W}_{H} .
\end{aligned}\right.
$$

Once $\left(\boldsymbol{u}_{H}, p_{H}\right)$ is obtained we get the solution of (4.1) by piecing together the coarse and fine components, i.e.,

$$
\left(\boldsymbol{u}_{H, \delta}, p_{H, \delta}\right)=\left(\boldsymbol{u}_{H}, p_{H}\right)+\left(\boldsymbol{u}_{\delta}\left(\boldsymbol{u}_{H}\right), p_{\delta}\left(\boldsymbol{u}_{H}\right)\right)+\left(\boldsymbol{u}_{\delta}\left(F_{m}\right), p_{\delta}\left(F_{m}\right)\right)
$$

The above construction results in Algorithm 1 for computing $\left(\boldsymbol{u}_{H, \delta}, p_{H, \delta}\right)$.

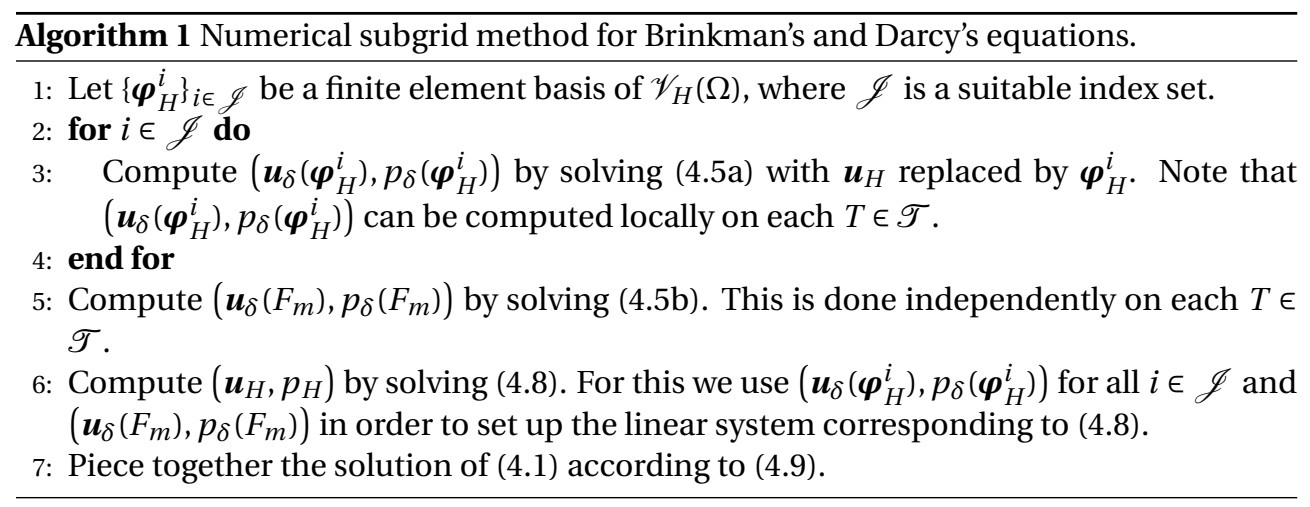

REMARK 4.2. We emphasize that Algorithm 1 is essentially a special way for computing $\left(\boldsymbol{u}_{H, \delta}, p_{H, \delta}\right)$ satisfying (4.1). 
5. Extending the Numerical Subgrid Method by Alternating Schwarz Iterations. As noted in the previous section Algorithm 1 is just some special way of computing the solution of (4.1), i.e., the finite element solution corresponding to the space $\left(\mathscr{V}_{H, \delta}, \mathscr{W}_{H, \delta}\right)$. As mentioned in Remark 2.1 the main difference between the spaces $\left(\mathscr{V}_{H, \delta}, \mathscr{W}_{H, \delta}\right)$ compared with $\left(H_{0}^{1}(\Omega)^{n}, L_{0}^{2}(\Omega)\right)$ and $\left(H_{0}(\operatorname{div} ; \Omega), L_{0}^{2}(\Omega)\right)$, respectively, is that the former only has some coarse degrees of freedom across coarse cell boundaries. Thus, any fine-scale features of the solution $(\boldsymbol{u}, p)$ across those coarse cell boundaries can only be captured poorly by functions in $\left(\mathscr{V}_{H, \delta}, \mathscr{W}_{H, \delta}\right)$. Algorithm 2 addresses this problem by performing alternating Schwarz iterations between the spaces $\left(\mathscr{V}_{H, \delta}, \mathscr{W}_{H, \delta}\right)$ and $\left(\mathscr{V}^{\tau}(\iota), \mathscr{W}^{\tau}(\iota)\right)$, with

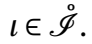

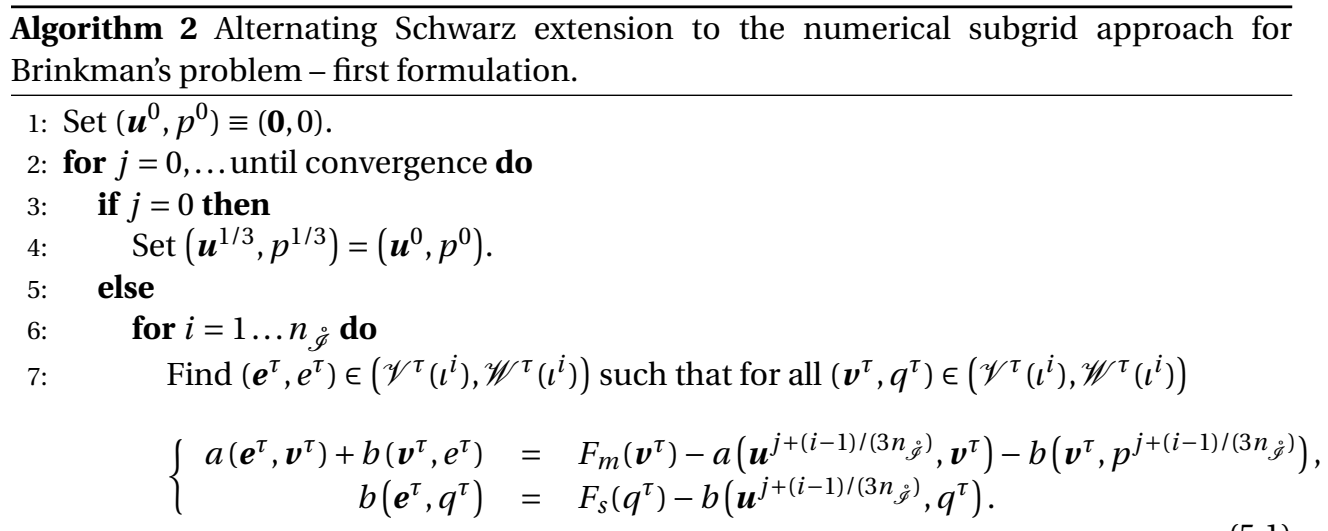

8

Set

$$
\left(\boldsymbol{u}^{j+i /\left(3 n_{\mathfrak{I}}\right)}, p^{j+i /\left(3 n_{\mathfrak{I}}\right)}\right)=\left(\boldsymbol{u}^{j+(i-1) /\left(3 n_{\mathfrak{I}}\right)}, p^{j+(i-1) /\left(3 n_{\mathfrak{I}}\right)}\right)+\left(\boldsymbol{e}^{\tau}, e^{\tau}\right),
$$

9:

10:

11:

12: $\quad$ Set

\section{end for \\ end if}

Find $\left(\boldsymbol{e}_{H, \delta}, e_{H, \delta}\right) \in\left(\mathscr{V}_{H, \delta}, \mathscr{W}_{H, \delta}\right)$ such that for all $\left(\boldsymbol{v}_{H, \delta}, q_{H, \delta}\right) \in\left(\mathscr{V}_{H, \delta}, \mathscr{W}_{H, \delta}\right)$ we have

$$
\left\{\begin{aligned}
a\left(\boldsymbol{e}_{H, \delta}, \boldsymbol{v}_{H, \delta}\right)+b\left(\boldsymbol{v}_{H, \delta}, e_{H, \delta}\right) & =F_{m}\left(\boldsymbol{v}_{H, \delta}\right)-a\left(\boldsymbol{u}^{j+1 / 3}, \boldsymbol{v}_{H, \delta}\right)-b\left(\boldsymbol{v}_{H, \delta}, p^{j+1 / 3}\right), \\
b\left(\boldsymbol{e}_{H, \delta}, q_{H, \delta}\right) & =F_{s}\left(q_{H, \delta}\right)-b\left(\boldsymbol{u}^{j+1 / 3}, q_{H, \delta}\right)
\end{aligned}\right.
$$

$$
\left(\boldsymbol{u}^{j+1}, p^{j+1}\right)=\left(\boldsymbol{u}^{j+1 / 3}, p^{j+1 / 3}\right)+\left(\boldsymbol{e}_{H, \delta}, e_{H, \delta}\right)
$$

13: end for

REMARK 5.1. It is straightforward to see that $\left(\boldsymbol{u}^{1}, p^{1}\right) \equiv\left(\boldsymbol{u}_{H, \delta}, p_{H, \delta}\right)$ solving (4.1).

Now, problem (5.3) is of exactly the same form as (4.1). Thus, by the same reasoning as in the previous section we may replace (5.3) by the following two problems:

Find $\left(\boldsymbol{e}_{\delta}, e_{\delta}\right) \in(\delta \mathscr{V}, \delta \mathscr{W})$ such that for all $\left(\boldsymbol{v}_{\delta}, q_{\delta}\right) \in(\delta \mathscr{V}, \delta \mathscr{W})$ we have

$$
\left\{\begin{aligned}
a\left(\boldsymbol{e}_{\delta}, \boldsymbol{v}_{\delta}\right)+b\left(\boldsymbol{v}_{\delta}, e_{\delta}\right) & =F_{m}\left(\boldsymbol{v}_{\delta}\right)-a\left(\boldsymbol{u}^{j+1 / 3}, \boldsymbol{v}_{\delta}\right)-b\left(\boldsymbol{v}_{\delta}, p^{j+1 / 3}\right) \\
b\left(\boldsymbol{e}_{\delta}, q_{\delta}\right) & =-b\left(\boldsymbol{u}^{j+1 / 3}, q_{\delta}\right)
\end{aligned}\right.
$$


Find $\left(\boldsymbol{e}_{H}, e_{H}\right) \in\left(\mathscr{V}_{H}, \mathscr{W}_{H}\right)$ such that for all $\left(\boldsymbol{v}_{H}, q_{H}\right) \in\left(\mathscr{V}_{H}, \mathscr{W}_{H}\right)$ we have

$$
\left\{\begin{aligned}
\widetilde{a}\left(\boldsymbol{e}_{H}, \boldsymbol{v}_{H}\right)+b\left(\boldsymbol{v}_{H}, e_{H}\right) & =F_{m}\left(\boldsymbol{v}_{H}\right)-a\left(\boldsymbol{u}^{j+1 / 3}+\boldsymbol{e}_{\delta}, \boldsymbol{v}_{H}\right)-b\left(\boldsymbol{v}_{H}, p^{j+1 / 3}\right), \\
b\left(\boldsymbol{e}_{H}, q_{H}\right) & =F_{S}\left(q_{H}\right)-b\left(\boldsymbol{u}^{j+1 / 3}, q_{H}\right) .
\end{aligned}\right.
$$

Here, (5.5a) and (5.5b) correspond to (4.5b) and (4.8), respectively, and analogous to (4.9) $\left(\boldsymbol{e}_{H, \delta}, e_{H, \delta}\right)$ from (5.3) is obtained by

$$
\left(\boldsymbol{e}_{H, \delta}, e_{H, \delta}\right)=\left(\boldsymbol{e}_{H}, e_{H}\right)+\left(\boldsymbol{u}_{\delta}\left(\boldsymbol{e}_{H}\right), p_{\delta}\left(\boldsymbol{e}_{H}\right)\right)+\left(\boldsymbol{e}_{\delta}, e_{\delta}\right)
$$

To obtain (5.5a) it is important to note that $F_{s}\left(q_{\delta}\right)=0$ due to (3.6), (3.4), (3.3), and (2.4).

Now, let us define

$$
\left(\boldsymbol{u}^{j+2 / 3}, p^{j+2 / 3}\right):=\left(\boldsymbol{u}^{j+1 / 3}, p^{j+1 / 3}\right)+\left(\boldsymbol{e}_{\delta}, e_{\delta}\right) .
$$

Combining this with (5.4) and (5.6) we obtain

$$
\left(\boldsymbol{u}^{j+1}, p^{j+1}\right)=\left(\boldsymbol{u}^{j+2 / 3}, p^{j+2 / 3}\right)+\left(\boldsymbol{e}_{H}, e_{H}\right)+\left(\boldsymbol{u}_{\delta}\left(\boldsymbol{e}_{H}\right), p_{\delta}\left(\boldsymbol{e}_{H}\right)\right)
$$

We furthermore observe that due to (2.4a) and (2.4b) we may simplify (5.5b) to obtain

$$
\left\{\begin{aligned}
\widetilde{a}\left(\boldsymbol{e}_{H}, \boldsymbol{v}_{H}\right)+b\left(\boldsymbol{v}_{H}, \boldsymbol{e}_{H}\right) & =F_{m}\left(\boldsymbol{v}_{H}\right)-a\left(\boldsymbol{u}^{j+2 / 3}, \boldsymbol{v}_{H}\right)-b\left(\boldsymbol{v}_{H}, p^{j+2 / 3}\right), \\
b\left(\boldsymbol{e}_{H}, q_{H}\right) & =F_{s}\left(q_{H}\right)-b\left(\boldsymbol{u}^{j+2 / 3}, q_{H}\right) .
\end{aligned}\right.
$$

Thus, we can rewrite Algorithm 2 in form of Algorithm 3, and we summarize our derivations in the following

Proposition 5.2. The iterates $\left(\boldsymbol{u}^{j}, p^{j}\right)$ of Algorithms 2 and 3 coincide.

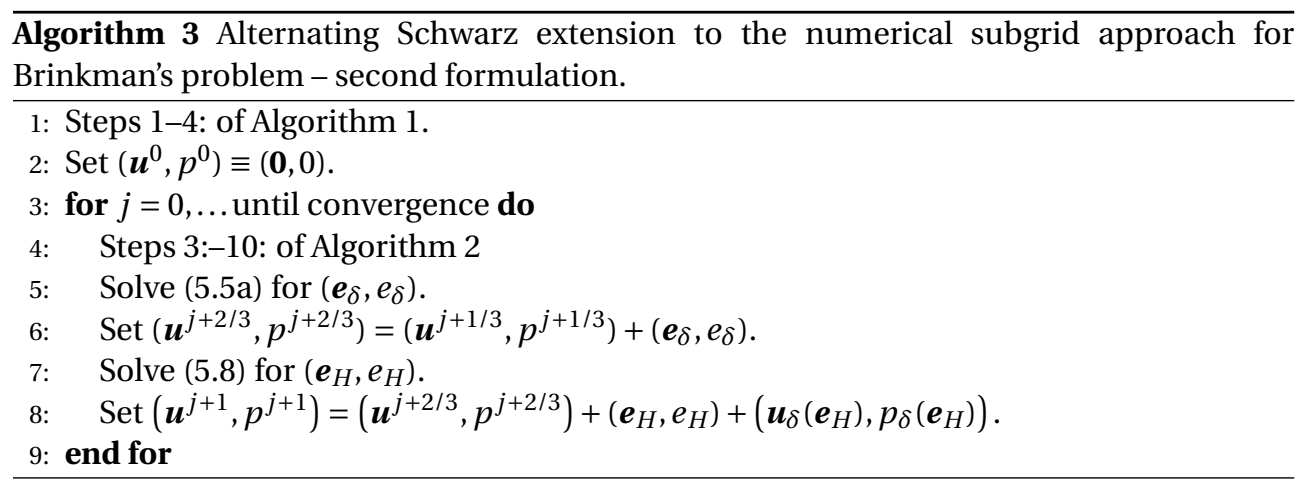

REMARK 5.3. Algorithm 3 also has a different interpretation than just being some equivalent formulation of Algorithm 2. It is easy to see that $\left(\boldsymbol{u}^{2 / 3}, p^{2 / 3}\right)=\left(\boldsymbol{u}_{\delta}\left(F_{m}\right), p_{\delta}\left(F_{m}\right)\right)$, i.e., $\left(\boldsymbol{u}^{2 / 3}, p^{2 / 3}\right)$ is the solution of (4.5b). For $j \geq 1\left(\boldsymbol{u}^{j+2 / 3}, p^{j+2 / 3}\right)$ is the solution of (4.5b) with the homogeneous boundary conditions being replaced by (in general) inhomogeneous ones defined by $\left(\boldsymbol{u}^{j+1 / 3}, p^{j+1 / 3}\right)$. Besides, (5.8) is of the same form as (4.8). Thus, Algorithm 3 can be viewed as a subgrid algorithm that iteratively improves the local boundary conditions of the response to the right hand side.

REMARK 5.4 (Solvability of (5.5a)). Looking at (5.5a) it is not immediately evident that the boundary conditions given by $\boldsymbol{u}^{j+1 / 3}$ are compatible, i.e., that

$$
\int_{\partial T} \boldsymbol{u}^{j+1 / 3} \cdot \boldsymbol{n} d s=0
$$


is satisfied for all $T \in \mathscr{T}$. If $\boldsymbol{u}^{j+1 / 3} \equiv \boldsymbol{u}$ solving (2.1) and (2.2), respectively, this condition certainly holds. For an arbitrary iterate $\boldsymbol{u}^{j+1 / 3}$ we, however, need to project the normal component of $\boldsymbol{u}^{j+1 / 3}$ at $\partial T$ in order to guarantee that (5.9) is satisfied. This is done in such a way that mass conservation is maintained in the entire domain. By a similar procedure we also ensure the solvability of (5.1). This procedure also allows to drop restriction (3.6), i.e., it is possible to treat boundary conditions with fine features in this iterative framework.

REMARK 5.5. As stated above Algorithm 2 (and equivalently Algorithm 3) is an alternating Schwarz iteration using the spaces $\left(\mathscr{V}_{H, \delta}, \mathscr{W}_{H, \delta}\right)$ and $\left(\mathscr{V}^{\tau}(\iota), \mathscr{W}^{\tau}(\iota)\right)$, with $\iota \in \mathscr{I}^{\circ}$. More precisely, in the terminology of [28] it is a multiplicative Schwarz iteration, with $\left(\mathscr{V}_{H, \delta}, \mathscr{W}_{H, \delta}\right)$ taking the role of the coarse space in [28]. By the reasoning in [28, Section 10.4.2] the analysis of alternating Schwarz methods for saddle point problems, like the one we consider, may be reduced to the standard case of elliptic problems. Thus, the standard convergence results (cf. [28, Section 2.5]) are applicable.

6. Numerical Results and Conclusions. In this section we investigate the performance of the methods developed above by means of a series of numerical examples. To make the above procedure fully computational we need to find a finite element analog of the spaces $\delta \mathscr{V}(T)$ and $\delta \mathscr{W}(T)$. For this each finite element $T$ is subdivided into a number of subelements with smaller step-size $h$. The nice property of this partition is that each element could have its own subgrid including the case when for some finite element $T$ the spaces $\delta \mathscr{V}(T)$ and $\delta \mathscr{W}(T)$ could be empty. For our numerical experiments on the fine mesh we have taken $\delta \mathscr{V}(T)$ to be the space of BDM1 finite elements (already described above) while the space $\delta \mathscr{W}(T)$ consists of piece-wise constant scalar functions with mean value zero on $T$. All of our numerical experiments were performed on a $128 \times 128$ square mesh, while the coarse meshes were $4 \times 4,8 \times 8$, and $16 \times 16$. This means that on each coarse grid element the corresponding spaces $\delta \mathscr{V}(T)$ and $\delta \mathscr{W}(T)$ are in fact finite element spaces defined on $32 \times 32,16 \times 16$, and $8 \times 8$ meshes, respectively.

The algorithms described above have been implemented in the open source software deal.II - a General Purpose Object Oriented Finite Element Library of Bangerth, Kanschat and Hartman, [6]. The library allows unified implementation of both two and threedimensional problems. However, our numerical experiments were performed on twodimensional examples only.

6.1. Objectives and Numerical Examples. In our numerical experiments we shall pursue the following objectives:

(1) Investigate the performance of Algorithm 1, i.e., the subgrid method (without Schwarz iterations). In particular, we are interested in finding the dependence of the accuracy with respect to the choice of $H$ and also with respect to the magnitude of variations in the permeability $\kappa$.

(2) Investigate the performance of Algorithm 3 (and equivalently Algorithm 2). This includes in particular a verification that the iterates converge to the reference solution computed on a global fine gird. We are furthermore interested in checking the dependence of this convergence on the choice of the mesh parameter $H$ and the magnitude of variations in $\kappa$.

For the achievement of these objectives we employ several examples motivated by practical situations outlined in the introduction. More precisely, we consider the following flow regimes and example geometries:

(a) Flow in a periodic geometry modeled by Darcy's equations - example geometry given in Figure 6.1(a). This example hardly has any meaningful physical interpretation, but is frequently considered in homogenization theory (cf. [19, 22]). 


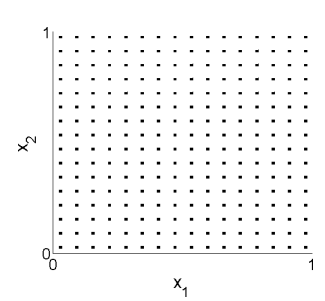

(a) Periodic geometry.

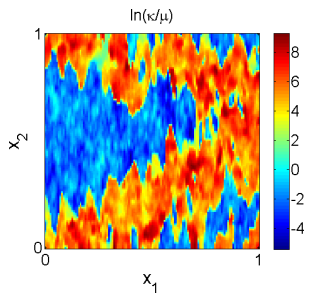

(b) SPE10 benchmark geometry.

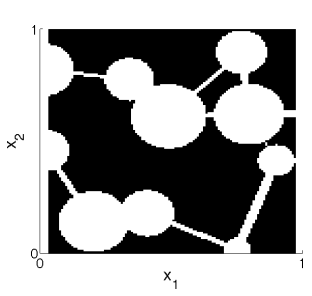

(c) Vuggy medium.

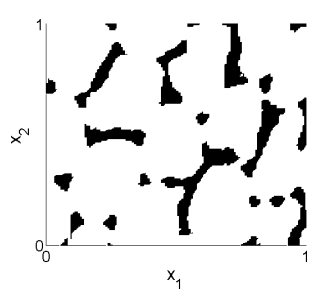

(d) Open foam.

FIG. 6.1. Different geometries with lowly (black and blue, respectively) and highly (white and red, respectively) permeable regions.

(b) Flow in a natural reservoir modeled by Darcy's equations - example geometry given in Figure 6.1(b). This geometry is the spatially rescaled slice 44 of the geometry of the Tenth SPE Comparative Solution Project (cf. [11]).

(c) Flow in a vuggy porous medium modeled by Brinkman's equations - example geometry given in Figure 6.1(c). This example is relevant to simulations in reservoirs with large cavities (cf. [31]).

(d) Flow in an open foam modeled by Brinkman's equations - example geometry given in Figure 6.1(d)). This example is relevant to filtration processes, heat exchangers, etc. (cf. $[27,35])$.

REMARK 6.1 (Comments on geometries in Figure 6.1). The black (blue) and white (red) areas in the geometries of Figure 6.1 denote the regions of low and high permeabilities, respectively. From an upscaling point of view, the periodic geometry can be considered the simplest of the four, since the length-scale of the lowly permeable inclusions is clearly separated from the length-scale defined by the size of the entire geometry. For the other three geometries such a clear separation of scales does not exist. As discussed in [41] non-local fine features usually entail large boundary layers, which are generally hard to capture by upscaling procedures.

We now specify the precise problem parameters for our numerical experiments. The enumeration of the numerical examples given below is to be understood as follows: "Example 1(1.ii)" refers to a problem setting as described in Example 1 with $\mu \kappa^{-1} \equiv 1 e-2$ in the white parts of the geometry (case (1) above), and $\mathscr{T}$ consisting of $8 \times 8$ uniform grid cells (case (ii) above).

EXAMPLE 1 (Darcy - periodic geometry).

$$
\boldsymbol{f}_{m} \equiv \mathbf{0}, \quad g \equiv\left[\begin{array}{l}
1 \\
0
\end{array}\right] \cdot \boldsymbol{n}, \quad \mu \kappa^{-1} \equiv 1 \text { e3 in black regions of Figure 6.1(a), and }
$$

(1) $\mu \kappa^{-1} \equiv 1 e-2, \quad$ (2) $\mu \kappa^{-1} \equiv 1, \quad$ (3) $\mu \kappa^{-1} \equiv 1 e 2 \quad$ in white region of Figure 6.1 (a);
(i) $\mathscr{T}$ a grid of $16^{2}$ cells
(ii) $\mathscr{T}$ a grid of $8^{2}$ cells
(iii) $\mathscr{T}$ a grid of $4^{2}$ cells.

EXAMPLE 2 (Darcy - SPE10 geometry).

$$
\boldsymbol{f}_{m} \equiv \mathbf{0}, \quad g \equiv\left[\begin{array}{l}
1 \\
0
\end{array}\right] \cdot \boldsymbol{n}, \quad \text { and } \mu \kappa^{-1} \text { according to Figure 6.1(b); }
$$


(i) $\mathscr{T}$ a grid of $16^{2}$ cells (ii) $\mathscr{T}$ a grid of $8^{2}$ cells (iii) $\mathscr{T}$ a grid of $4^{2}$ cells.

EXAMPLE 3 (Brinkman - vuggy medium).

$$
\begin{gathered}
\boldsymbol{f}_{m} \equiv \mathbf{0}, \quad \mathbf{g} \equiv\left[\begin{array}{l}
1 \\
0
\end{array}\right], \quad \mu \equiv 1 e-2, \quad \kappa \equiv 1 e-5 \text { in black regions of Figure } 6.1(c), \text { and } \\
\begin{array}{llll}
\text { (1) } \kappa \equiv 1, \quad(2) \kappa \equiv 1 e-2, & \text { (3) } \kappa \equiv 1 e-4 \text { in white regions of Figure } 6.1(c) . \\
\text { (i) } \mathscr{T} \text { a grid of } 16^{2} \text { cells } & \text { (ii) } \mathscr{T} \text { a grid of } 8^{2} \text { cells } \quad \text { (iii) } \mathscr{T} \text { a grid of } 4^{2} \text { cells. }
\end{array}
\end{gathered}
$$

EXAMPLE 4 (Brinkman - open foam).

$$
\begin{gathered}
\boldsymbol{f}_{m} \equiv \mathbf{0}, \quad \mathbf{g} \equiv\left[\begin{array}{l}
1 \\
0
\end{array}\right], \quad \mu \equiv 1 e-2, \quad \kappa \equiv 1 e-5 \text { in black regions of Figure } 6.1(d), \text { and } \\
\begin{array}{llll}
\text { (1) } \kappa \equiv 1, & \text { (2) } \kappa \equiv 1 e-2, & \text { (3) } \kappa \equiv 1 e-4 \text { in white regions of Figure } 6.1(d) . \\
\text { (i) } \mathscr{T} \text { a grid of } 16^{2} \text { cells } & \text { (ii) } \mathscr{T} \text { a grid of } 8^{2} \text { cells } \quad \text { (iii) } \mathscr{T} \text { a grid of } 4^{2} \text { cells. }
\end{array}
\end{gathered}
$$

For Examples 1-4 we choose $\Omega=(0,1)^{2}$ and whenever Algorithm 3 is applied we set $C_{l}$ determining the size of the overlapping region to be $\frac{1}{4}$. Also, for the discretization of (2.1) we choose $\alpha=20$ in (3.2). The reference solutions are obtained by solving discretizations on a grid of $128 \times 128$ uniform cells, and, as stated above, all local fine computations are performed on the restriction of this global fine mesh to the respective subdomains.

Having defined Examples 1-4 we can now investigate our two objectives.

6.2. Performance of Algorithm 1. For clarity we again note that by Remark 5.1 the first iterate, i.e., $\left(\boldsymbol{u}^{1}, p^{1}\right)$, of Algorithm 3 is equal to the result of the subgrid Algorithm 1. Furthermore, recall that $\left(\boldsymbol{u}^{1}, p^{1}\right)$, by definition, cannot approximate the full fine scale solution, due to the imposed localization conditions on the interfaces between the coarse cells. However, the solution $\left(\boldsymbol{u}^{1}, p^{1}\right)$, in addition to the coarse scale information, contains a lot of the features of the fine solution, and therefore a comparison with the fine scale solution is of interest.

Table 6.1 summarizes the results by reporting the relative errors for the velocity with respect to the reference solutions. Analyzing this data we can make the following observations:

Dependence on $\kappa$. We see that for all considered instances larger jumps in $\kappa$ lead to larger errors. This is not very surprising, since increasing jumps in $k$ generally leads to more pronounced features in the solution, which are increasingly harder to resolve by functions in $\left(\mathscr{V}_{H, \delta}, \mathscr{W}_{H, \delta}\right)$ compared to $\left(H_{0}^{1}(\Omega)^{n}, L_{0}^{2}(\Omega)\right)$ and $\left(H_{0}(\operatorname{div} ; \Omega), L_{0}^{2}(\Omega)\right)$, respectively. 
Dependence on $H$. Considering different choices of $H$, we cannot draw a clear conclusion. For the periodic geometry, i.e., Example 1, increasing $H$ by a factor of 2 yields pronounced decreases in the errors. The errors in the velocity are approximately reduced by a factor of 1.5. This behavior can be explained by the estimates in [4, Theorem 6.1] if the error term $\sqrt{\epsilon / H}$ is dominating, where $\epsilon$ denotes the periodicity length.

On the other hand, for Examples 2-4, which have a much more complicated internal structure, $H$ is expected to influence the accuracy of the subgrid solution in a more complicated way. In our simulations, the observed changes in the errors are rather small and non-uniform, i.e., some of the errors decrease/increase with increasing $H$. A more detailed study of the dependence of the two-scale solution on $H$ is not a main target of this paper and will be analyzed and discussed separately in the future.

Quality of the approximation. Considering the magnitudes of the relative errors reported in Table 6.1 we can say that depending on the geometry and the targeted application they may still be acceptable. In particular for the examples with moderate jumps in $\kappa$ the relative errors are in the range of $10 \%$. In many practical situations the relevant problem parameters, such as the shape of the geometry, the values of $\kappa$, etc., are only given up to a certain accuracy. It is not unusual that these uncertainties entail an uncertainty in the solution, which can easily exceed $10 \%$. In these situations it would therefore be a waste of resources to compute very accurate solutions based on inaccurate data. For these instances the numerical subgrid method may be a valuable tool for computing approximate solutions of (2.1) and (2.2).

In Figures 6.2 and 6.3 we also provide two plots of the first velocity component, i.e., $u_{1}$, of reference solutions ( $\left.\boldsymbol{u}_{\text {ref }}, p_{\text {ref }}\right)$ and some selected solutions of Algorithm 1 corresponding to the examples above with different choices of $H$. Comparing these plots we see that in many cases the subgrid solutions actually look rather similar to the reference ones. One striking difference, however, are the jumps in the subgrid solutions that are aligned with the coarse cell boundaries. These jumps are, of course, due to the lack of fine degrees of freedom across coarse edges and well understood in the multiscale finite element analysis (e.g. $[41,15])$.

In Figure 6.4 we provide a plot of the pressure for Example 3(1.ii). It can be seen from Table 6.1 and from the plot, that the errors of the pressure are quite large for a $4 \times 4$ coarse mesh. However, the error improves substantially if a $16 \times 16$ coarse mesh is used. In conclusion, we see that keeping a right balance between the number of coarse and fine grid cells, and also balancing this with the accuracy of the input data, we can ensure accuracies acceptable for the engineering practice using meshes of reasonable sizes.

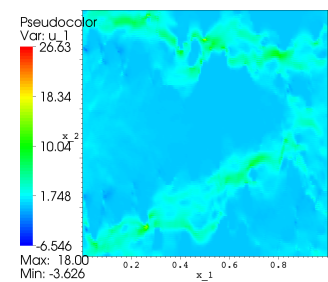

(a) Reference solution

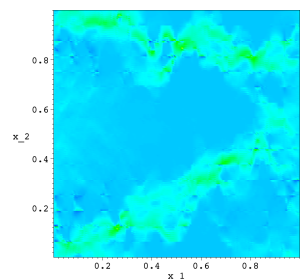

(b) $H=1 / 16$.

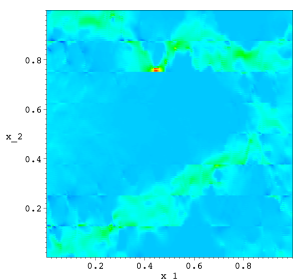

(c) $H=1 / 8$

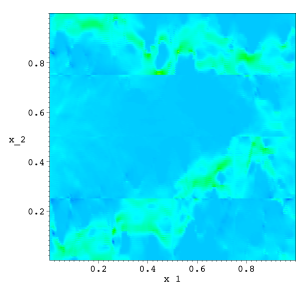

(d) $H=1 / 4$.

FIG. 6.2. First velocity component, $u_{1}$, corresponding to Example 2 for the reference solution (computed on the global fine grid) and the subgrid solution computed on different coarse grids. 


\begin{tabular}{|c|c|c|c|c|c|c|c|}
\cline { 2 - 8 } \multicolumn{1}{c|}{} & \multicolumn{2}{c|}{ Velocity } & \multicolumn{3}{c|}{ Pressure } \\
\cline { 2 - 8 } \multicolumn{1}{c|}{} & Contrast & $1 / 16$ & $1 / 8$ & $1 / 4$ & $1 / 16$ & $1 / 8$ & $1 / 4$ \\
\hline \multirow{3}{*}{ Example 1 } & $1 \mathrm{e} 5$ & $3.18 \mathrm{e}-02$ & $2.18 \mathrm{e}-02$ & $1.42 \mathrm{e}-02$ & $1.28 \mathrm{e}-03$ & $7.16 \mathrm{e}-04$ & $4.05 \mathrm{e}-04$ \\
\cline { 2 - 8 } & $1 \mathrm{l} 3$ & $3.17 \mathrm{e}-02$ & $2.17 \mathrm{e}-02$ & $1.42 \mathrm{e}-02$ & $1.27 \mathrm{e}-03$ & $7.13 \mathrm{e}-04$ & $4.04 \mathrm{e}-04$ \\
\cline { 2 - 8 } & $1 \mathrm{e} 1$ & $2.48 \mathrm{e}-02$ & $1.70 \mathrm{e}-02$ & $1.11 \mathrm{e}-02$ & $8.38 \mathrm{e}-04$ & $4.90 \mathrm{e}-04$ & $2.88 \mathrm{e}-04$ \\
\hline Example 2 & $2.54 \mathrm{e}+06$ & $3.19 \mathrm{e}-01$ & $4.95 \mathrm{e}-01$ & $3.70 \mathrm{e}-01$ & $2.05 \mathrm{e}-01$ & $3.31 \mathrm{e}-01$ & $5.41 \mathrm{e}-01$ \\
\hline \multirow{3}{*}{ Example 3 } & $1 \mathrm{e} 5$ & $2.71 \mathrm{e}-01$ & $2.82 \mathrm{e}-01$ & $3.00 \mathrm{e}-01$ & $1.56 \mathrm{e}-01$ & $2.15 \mathrm{e}-01$ & $2.70 \mathrm{e}-01$ \\
\cline { 2 - 8 } & $1 \mathrm{l} 3$ & $2.69 \mathrm{e}-01$ & $2.80 \mathrm{e}-01$ & $2.94 \mathrm{e}-01$ & $1.54 \mathrm{e}-01$ & $2.13 \mathrm{e}-01$ & $2.68 \mathrm{e}-01$ \\
\hline \multirow{3}{*}{ Example 4 } & $1 \mathrm{e} 1$ & $1.62 \mathrm{e}-01$ & $1.75 \mathrm{e}-01$ & $1.67 \mathrm{e}-01$ & $7.10 \mathrm{e}-02$ & $1.12 \mathrm{e}-01$ & $1.57 \mathrm{e}-01$ \\
\cline { 2 - 8 } & $1 \mathrm{e} 5$ & $2.45 \mathrm{e}-01$ & $3.31 \mathrm{e}-01$ & $3.71 \mathrm{e}-01$ & $6.18 \mathrm{e}-01$ & $1.19 \mathrm{e}+00$ & $1.55 \mathrm{e}+00$ \\
\cline { 2 - 8 } & $1 \mathrm{l} 3$ & $2.41 \mathrm{e}-01$ & $3.28 \mathrm{e}-01$ & $3.69 \mathrm{e}-01$ & $5.82 \mathrm{e}-01$ & $1.12 \mathrm{e}+00$ & $1.46 \mathrm{e}+00$ \\
\hline
\end{tabular}

TABLE 6.1

Relative $L^{2}$-velocity and pressure errors for the numerical subgrid algorithm applied to Examples 1-4.

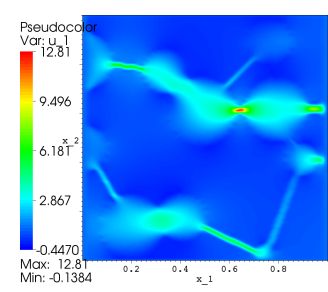

(a) Reference solution.

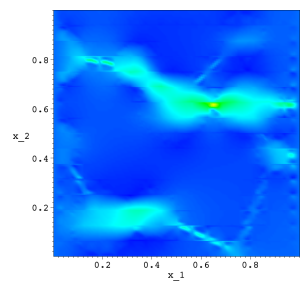

(b) $H=1 / 16$.

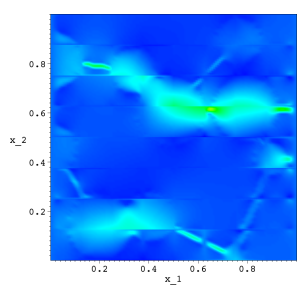

(c) $H=1 / 8$.

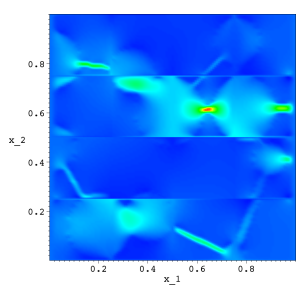

(d) $H=1 / 4$.

FIG. 6.3. First velocity component, $u_{1}$, corresponding to Example 3 for the reference solution (computed on the global fine grid) and the subgrid solution computed on different coarse grids.

6.3. Performance of Algorithm 3 (and Algorithm 2). We now discuss the performance of Algorithm 3. Figures 6.5-6.8 show the relative velocity and pressure errors for the first 39 iterations of Algorithm 3 after the initial subgrid solve for Examples 1-4.

Analyzing this data we can make the following observations:

Convergence to reference solution. The plots in Figures Figures 6.5-6.8 show the convergence of Algorithm 3.

For practical purposes it is, furthermore, important to note that the observed convergence is rather rapid at the beginning of the iterative process. In fact, in the discussed examples the error drops very quickly during the first iterations and then decreases linearly until the method has converged. The steep initial drop is particularly interesting for applications requiring only moderate degrees of accuracy, since in these cases a few iterations are enough to be sufficiently close to the reference solutions.

As mentioned above, the first iterate, which is the solution of the subgrid method, displays a crude representation of fine velocity features across coarse cell boundaries (see Figures $6.10(\mathrm{~b})-6.12(\mathrm{~b})$ ). However, after only a few iterations this deficiency is essentially resolved (see Figures 6.10(c)-6.12(c)). In fact, after only 5 iterations the iterative subgrid solutions are visually indistinguishable from their respective reference solutions. In addition to the reduction of the errors depicted in Figures 6.5-6.8 this is another very clear demonstration of the usefulness of our iterations and once again clarifies the interpretation of Algorithm 3 as given in Remark 5.3. 


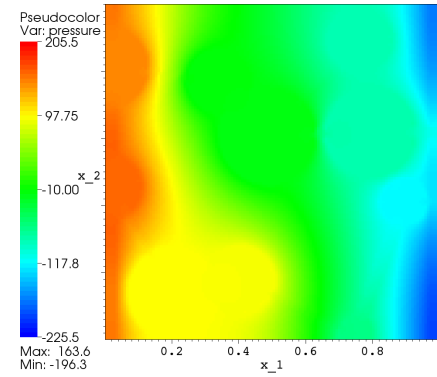

(a) Reference solution.

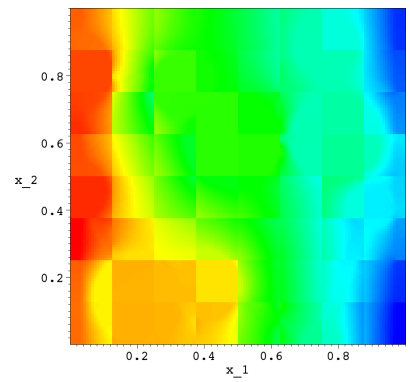

(b) Subgrid solution.

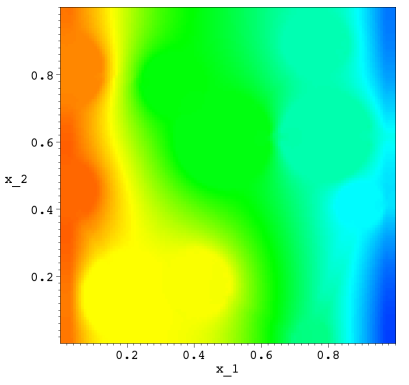

(c) Iterated subgrid solution.

FIG. 6.4. Pressure component, $p$, corresponding to Example 3(1.ii) for the reference solution computed on the global fine $128 \times 128$ grid, the subgrid solution computed on the coarse $8 \times 8$ grid (without any iterations), and the iterated subgrid solution after 5 iterations.

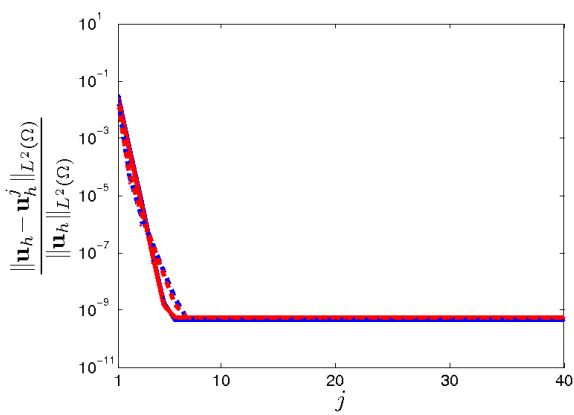

(a) Velocity error.

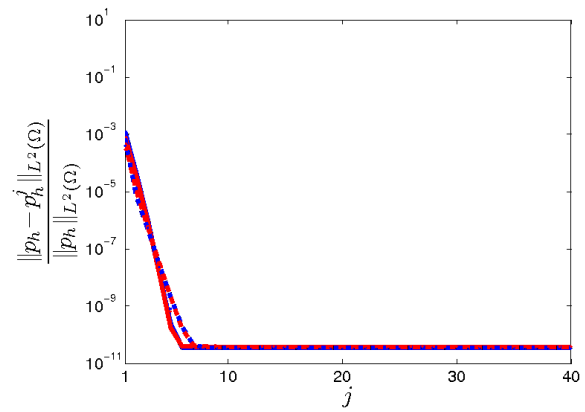

(b) Pressure error.

FIG. 6.5. Relative errors for Example 1. Solid line (-): $H=1 / 16$, dashed line (- -): $H=1 / 8$, dotted line ( $\cdots$ ): $H=1 / 4$, black: contrast $=1 e 5$, blue: contrast $=1 e 3$, red: contrast $=1 e 1$.

Dependence on $\kappa$. The magnitude of variations in $\kappa$ influences Algorithm 3 in a similar way as it influences Algorithm 1. The convergence rate of the two-level domain decomposition method, i.e., Algorithm 3, decreases with increasing the magnitude of the variations. This observation is in particular true for those examples whose solutions display fine velocity features across coarse cell boundaries. For the periodic geometry there are hardly any of those features. This is why in this case Algorithm 3 performs essentially independently of variations in $\kappa$ (see Figure 6.5). In general, it is expected that the convergence rate is less sensitive to $\kappa$ if long range correlations in $\kappa$ (if any) are entirely in the interior of individual coarse cells.

Dependence on $H$. As for Algorithm 1 the dependence of the convergence rate of Algorithm 3 on $H$ is non-uniform. However, variations of $H$ may greatly affect the convergence rate. The influence of $H$ on the convergence of domain decomposition methods for equations with smooth coefficients is well studied (cf. [28]). We expect that the observed inconsistent influence of $H$ on the rates of convergence reflects the fact that in our examples the coarse space approximation is not consistently improving with increased $H$.

Summing up, we conclude that we have developed a numerical subgrid algorithm for Brinkman's problem, summarized in Algorithm 1, using a discontinuous Galerkin discretization. This algorithm may serve as a useful numerical upscaling procedure. In particular, it is applicable to practical situations where only a moderate degree of accuracy is 


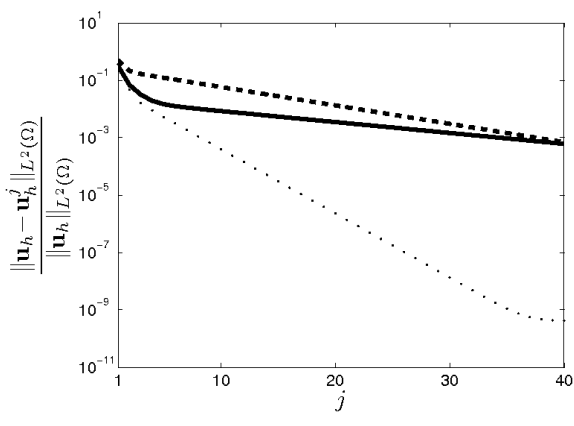

(a) Velocity error.

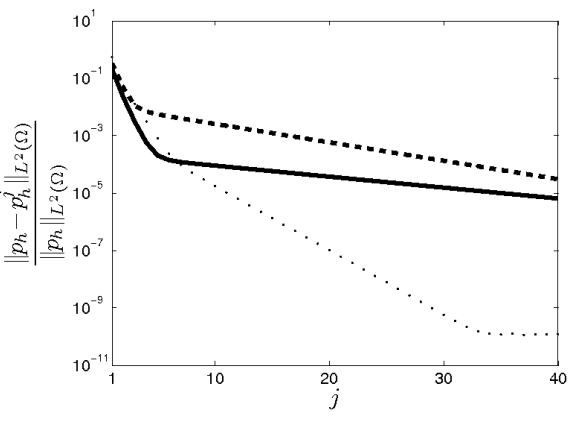

(b) Pressure error.

FIG. 6.6. Relative errors for Example 2. Solid line (-): $H=1 / 16$, dashed line (- -): $H=1 / 8$, dotted line ( $\cdots)$ : $H=1 / 4$.

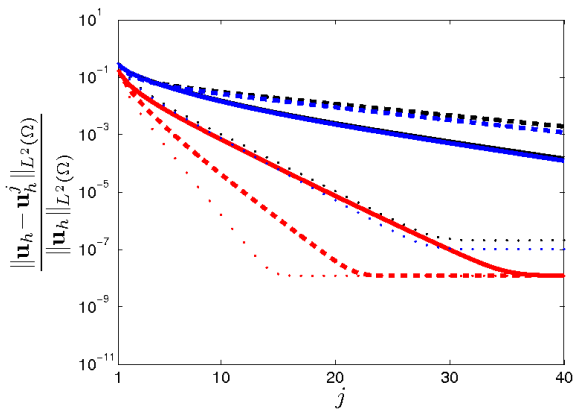

(a) Velocity error.

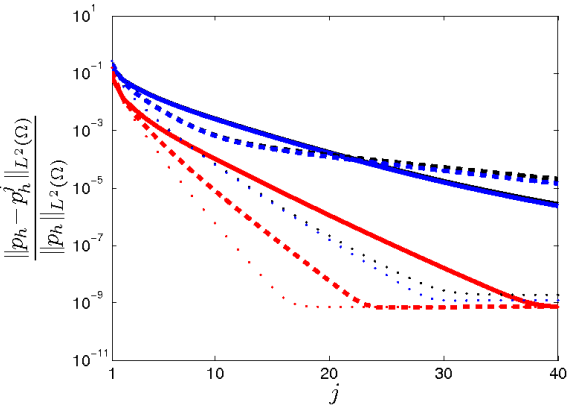

(b) Pressure error.

FIG. 6.7. Relative errors for Example 3. Solid line (-): $H=1 / 16$, dashed line (- -): $H=1 / 8$, dotted line ( $\cdots)$ : $H=1 / 4$, black: contrast $=1 e 5$, blue: contrast $=1 e 3$, red: contrast $=1 e 1$.

required and/or feasible to attain (due to uncertainties in the input data). We have furthermore introduced a two-scale iterative domain decomposition algorithm, i.e., Algorithm 3, for solving Darcy's and Brinkman's problem. This algorithm is an extension of the subgrid Algorithm 1, and ensures convergence to the solution of the global fine discretization. The developed algorithms require: (1) the solution of coarse global problem and (2) mutually independent fine local problems. This makes all algorithms very suitable for parallelization.

Acknowledgments. The research of O. Iliev was supported by DFG Project "Multiscale analysis of two-phase flow in porous mediawith complex heterogeneities". R. Lazarov has been supported by award KUS-C1-016-04, made by KAUST, made by King Abdullah University of Science and Technology (KAUST), by NSF Grant DMS-0713829. J. Willems was supported by DAAD-PPP D/07/10578, NSF Grant DMS-0713829, and the Studienstiftung des deutschen Volkes (German National Academic Foundation).

The authors express sincere thanks to Dr. Yalchin Efendiev for his valuable comments and numerous discussion on the subject of this paper. 


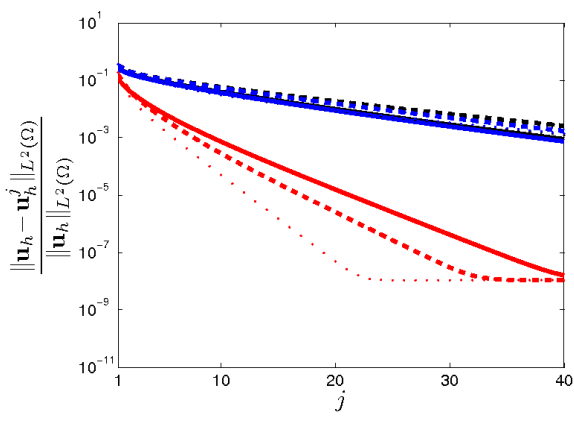

(a) Velocity error.

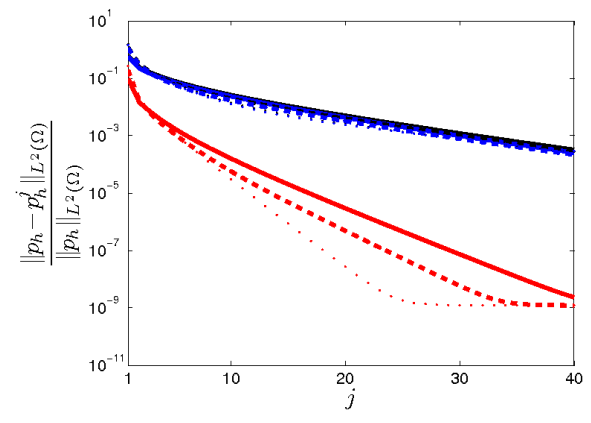

(b) Pressure error.

FIG. 6.8. Relative errors for Example 4. Solid line (-): $H=1 / 16$, dashed line (- -): $H=1 / 8$, dotted line ( $\cdots)$ : $H=1 / 4$, black: contrast $=1 e 5$, blue: contrast $=1 e 3$, red: contrast $=1 e 1$.

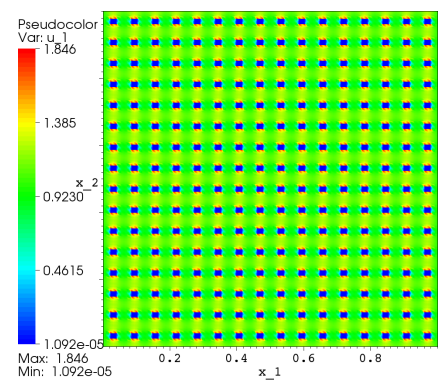

(a) Reference solution.

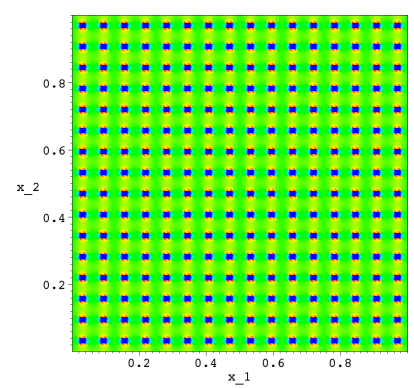

(b) Subgrid solution.

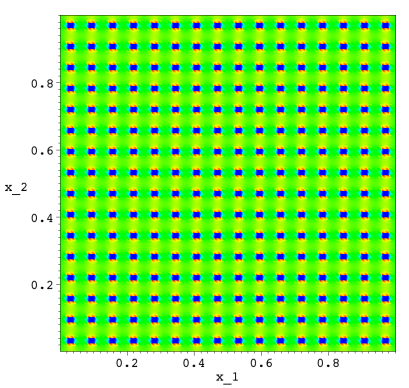

(c) Iterated subgrid solution.

FIG. 6.9. First velocity component, $u_{1}$, corresponding to Example 1(1.ii) for the reference solution (computed on the global fine grid), the subgrid solution (without any iterations), and the iterated subgrid solution after 5 iterations.

[1] G. Allaire. Homogenization of the Navier-Stokes equations in open sets perforated with tiny holes. I: Abstract framework, a volume distribution of holes. Arch. Ration. Mech. Anal., 113(3):209-259, 1991.

[2] P. Angot. Analysis of singular perturbations on the Brinkman problem for fictitious domain models of viscous flows. Math. Methods Appl. Sci., 22(16):1395-1412, 1999.

[3] T. Arbogast. Analysis of a two-scale, locally conservative subgrid upscaling for elliptic problems. SIAM J. Numer. Anal., 42(2):576-598, 2004.

[4] T. Arbogast and K. Boyd. Subgrid upscaling and mixed multiscale finite elements. SIAM J. Numer. Anal., 44(3):1150-1171, 2006.

[5] T. Arbogast and H. L. Lehr. Homogenization of a Darcy-Stokes system modeling vuggy porous media. Comput. Geosciences, 10(2):291-302, 2006.

[6] W. Bangerth, R. Hartmann, and G. Kanschat. deal.II - a general purpose object oriented finite element library. ACM Trans. Math. Softw., 33(4):24/1-24/27, 2007.

[7] J. Bear and Y. Bachmat. Introduction to Modeling of Transport Phenomena in Porous Media. Kluver Acedemic Publishers, Dordrecht, Netherlands, 1990.

[8] F. Brezzi and M. Fortin. Mixed and Hybrid Finite Element Methods, volume 15 of Springer Series in Computational Mathematics. Springer, 1st edition, 1991.

[9] H. C. Brinkman. A calculation of the viscouse force exerted by a flowing fluid on a dense swarm of particles. Appl. Sci. Res., A1:27-34, 1947.

[10] A. N. Bugrov and S. Smagulov. The fictitious regions method in boundary value problems for Navier-Stokes equations. Mathematical Models of Fluid Flows (Russian), pp. 79 - 90, 1978.

[11] M.A. Christie and M.J. Blunt. Tenth SPE comparative solution project: A comparison of upscaling techniques. SPE Res. Eng. Eval., 4:308-317, 2001.

[12] M. Dauge. Elliptic Boundary Value Problems in Corner Domains - Smoothness and Asymptotics ofSolutions. Lecture Notes in Mathematics 1341. Springer-Verlag, Berlin, 1988. 


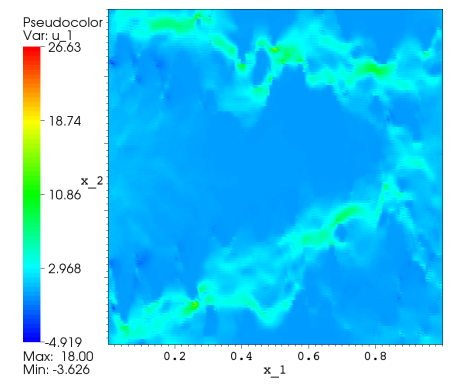

(a) Reference solution.

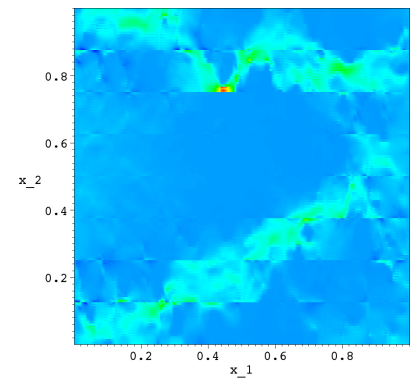

(b) Subgrid solution.

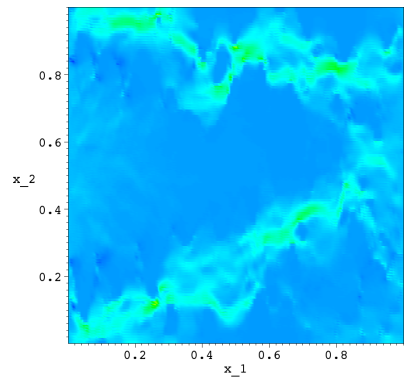

(c) Iterated subgrid solution.

FIG. 6.10. First velocity component, $u_{1}$, corresponding to Example 2(ii) for the reference solution (computed on the global fine grid), the subgrid solution (without any iterations), and the iterated subgrid solution after 5 iterations.

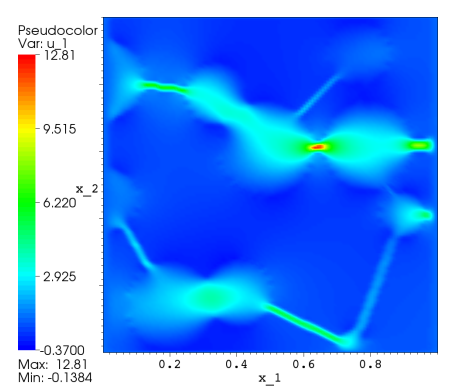

(a) Reference solution.

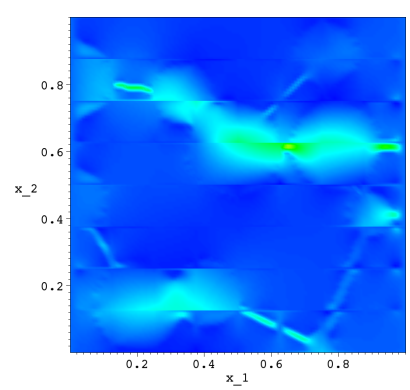

(b) Subgrid solution

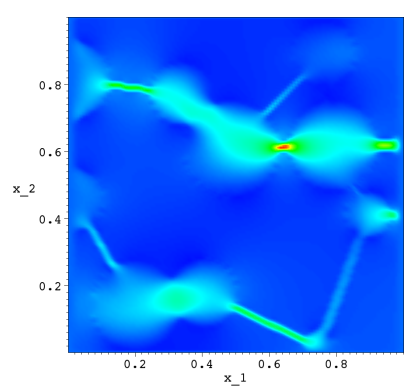

(c) Iterated subgrid solution.

FIG. 6.11. First velocity component, $u_{1}$, corresponding to Example 3(1.ii) for the reference solution (computed on the global fine grid), the subgrid solution (without any iterations), and the iterated subgrid solution after 5 iterations.

[13] Y. R. Efendiev, J. Galvis, and P. S. Vassilevski. Spectral element agglomerate algebraic multigrid methods for elliptic problems with high-contrast coefficients. Technical Report ISC-09-01, Institute for Scientific Computation, Texas A\& M University, 2009.

[14] Y. R. Efendiev, J. Galvis, and X. H. Wu. Multiscale finite element and domain decomposition methods for high-contrast problems using local spectral basis functions. Technical Report ISC-09-05, Institute for Scientific Computation, Texas A\& M University, 2009.

[15] Y. R. Efendiev and T. Hou. Multiscale Finite Element Methods. Theory and Applications. Springer, 2009.

[16] R. Glowinski, T-W. Pan, and J. Périaux. A fictitious domain method for external incompressible viscous flow modeled by Navier-Stokes equations. Comp. Meth. Appl. Mech. Engng., 112:133 - 148, 1994.

[17] P. Grisvard. Boundary Value Problems in Non-Smooth Domains. Pitman, London, 1985.

[18] A. Hannukainen, M. Juntunen, J. Könnö, and R. Stenberg. Finite element methods for the Brinkman problem. Talk at Center for Subsurface Modeling Affiliates Meeting, Helsinki University of Technology, October 14-15, 2009.

[19] U. Hornung, editor. Homogenization and Porous Media, volume 6 of Interdisciplinary Applied Mathematics. Springer, lst edition, 1997.

[20] O. P. Iliev, R. D. Lazarov, and J. Willems. Discontinuous Galerkin subgrid finite element method for approximation of heterogeneous Brinkman's equations. In Large-Scale Scientific Computing, volume 5910 of Lecture Notes in Comput. Sci., pages 14-25. Springer-Verlag, Berlin, Heidelberg, 2010.

[21] W. Jäger and A. Mikelic. On the boundary conditions at the contact interface between a porous medium and a free fluid. Annali della Scuola Normale Superiore di Pisa, Vol 23:403-465, 1996.

[22] V. V. Jikov, S. M. Kozlov, and O. A. Oleinik. Homogenization of Differential Operators and Integral Functionals. Springer, 1st edition, 1994.

[23] M. Kaviany. Principles of Heat Transfer in Porous Media. Springer-Verlag, New York, 1991.

[24] K. Khadra, P. Angot, S. Parneix, and J.-P. Caltagirone. Fictitious domain approach for numerical modelling 


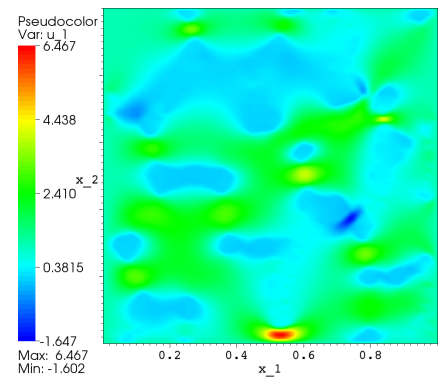

(a) Reference solution.

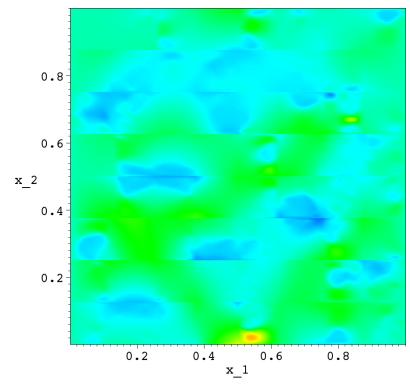

(b) Subgrid solution.

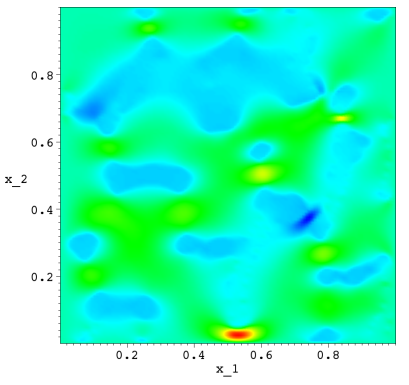

(c) Iterated subgrid solution.

FIG. 6.12. First velocity component, $u_{1}$, corresponding to Example 4(1.ii) for the reference solution (computed on the global fine grid), the subgrid solution (without any iterations), and the iterated subgrid solution after 5 iterations.

of Navier-Stokes equations. International Journal for Numerical Methods in Fluids, 34:651-684, 2000.

[25] A. N. Konovalov. The fictitious regions method in problems of mathematical physics. Computing methods in applied sciences and enginering, Proc. 4th int. Symp., Versailles 1979, pp. 29-40, 1980.

[26] W. J. Layton, F. Schieweck, and I. Yotov. Coupling fluid flow with porous media flow. SIAM J. Numer. Anal., 40(6):2195-2218, 2002.

[27] L.-P. Lefebvre, J.B. Banhart, and D.C. Dunand. Porous metals and metallic foams: Current status and recent developments. Advanced Engineering Materials, 10(9):775-787, 2008.

[28] T. P. A. Mathew. Domain Decomposition Methods for the Numerical Solution of Partial Differential Equations. Lecture Notes in Computational Science and Engineering. Springer, Berlin Heidelberg, 2008.

[29] J. Nolen, G. Papanicolaou, and O. Pironneau. A framework for adaptive multiscale methods for elliptic problems. Multiscale Model. Simul., 7(1):171-196, 2008.

[30] J.A. Ochoa-Tapia and S. Whitaker. Momentum transfer at the boundary between a porous medium and a homogeneous fluid. I. Theoretical development. Int. J. Heat Mass Transfer, 38:2635-2646, 1995.

[31] P. Popov, L. Bi, Y. Efendiev, R. Ewing, G. Qin, and J. Li. Multi-physics and multi-scale methods for modeling fluid flows through naturally fractured vuggy carbonate reservoirs. 15th SPE Middle East Oil \& Gas Show and Conference, Kingdom of Bahrain, 11-14 March, 2007, 2007. SPE 105378.

[32] K.R. Rajagopal. On a hierarchy of approximate models for flows of incompressible fluids through porous solids. Math. Models Methods Appl. Sci., 17(2):215-252, 2007.

[33] A.A. Samarskii, P.N. Vabishchevich, O.P. Iliev, and A.G. Churbanov. Numerical simulation of convection/diffusion phase change problems - a review. Int. J. Heat Mass Transfer, 36(17):4095-4106, 1993.

[34] D. Schötzau, Ch. Schwab, and A. Toselli. Mixed $h p$-DGFEM for incompressible flows. SIAM J. Numer. Anal., 40(6):2171-2194, 2003.

[35] M.V. Twigg and J.T. Richardson. Fundamentals and applications of structured ceramic foam catalysts. Ind. Eng. Chem. Res, 46:4166-4177, 2007.

[36] P. N. Vabishchevich. The method of fictitious domains in problems of mathematical physics. Moscow State University Publishing House (Russian), 158 pages, Moscow, 1991.

[37] J. Van lent, R. Scheichl, and I. G. Graham. Energy-minimizing coarse spaces for two-level Schwarz methods for multiscale PDEs. Numer. Linear Algebra Appl., 16(10):775-799, 2009.

[38] P. S. Vassilevski. General constrained energy minimization interpolation mapping for AMG. Siam J. Sci. Comp., 32:1 - 13, 2010.

[39] J. Wang and X. Ye. New finite element methods in computational fluid dynamics by H(div) elements. SIAM J. Numer. Anal., 45(3):1269-1286, 2007.

[40] J. Willems. Numerical Upscaling for Multiscale Flow Problems - Analysis and Algorithms. Suedwestdeutscher Verlag fuer Hochschulschriften, 2009.

[41] X. H. Wu, Y. Efendiev, and T. Y. Hou. Analysis of upscaling absolute permeability. Discrete Contin. Dyn. Syst., Ser. B, 2(2):185-204, 2002.

[42] J. C. Xu and L. T. Zikatanov. On an energy minimizing basis for algebraic multigrid methods. Comput. Vis. Sci., 7(3-4):121-127, 2004. 